\title{
World sheet diffeomorphisms and the canonical string
}

\author{
K. V. Kuchar and C. G. Torre ${ }^{\text {a) }}$ \\ Department of Physics, University of Utah, Salt Lake City, Utah 84112
}

(Received 8 February 1989; accepted for publication 5 April 1989)

\begin{abstract}
The degree to which the phase space of a closed bosonic string carries a representation of the two-dimensional diffeomorphism group $\operatorname{Diff}(M)$ is investigated. In particular, homomorphic mappings from the associated Lie algebra $\operatorname{diff}(M)$ into the Poisson algebra of functions on three natural phase spaces associated with the string are constructed. Two of these spaces are extended phase spaces based on the conformal and harmonic gauges, respectively. The third space is essentially the original phase space; the homomorphism in this case relies on the validity of the light-cone gauge. Homomorphisms from $\operatorname{diff}(M)$ into the extended phase spaces of the Batalin-Fradkin-Vilkovisky formalism are also constructed. While the methods used to represent $\operatorname{diff}(\boldsymbol{M})$ cannot be extended to represent all of $\operatorname{Diff}(\boldsymbol{M})$, the phase spaces $d o$ carry a representation of the subgroup of conformal isometries. It is argued that this subgroup is sufficiently large to serve as the dynamical group for the string. The implications of this work for a true Dirac quantization of the string via operator representations of $\operatorname{diff}(M)$ are discussed.
\end{abstract}

\section{INTRODUCTION}

It is well known that the presence of an infinite-dimensional invariance group for a dynamical system manifests itself through the appearance of constraints in the Hamiltonian formalism. ${ }^{1}$ For gauge theory, the constraint functions are linear in the canonical momenta and serve as comoments for the action of the gauge group on the phase space, that is, the Poisson algebra of the constraint functions is a homomorphic image of the Lie algebra of the gauge group. When the invariance group is the diffeomorphism group $\operatorname{Diff}(\boldsymbol{M})$ for the underlying space-time arena $M$, one finds a Hamiltonian constraint quadratic in momenta and, for field theories, a momentum constraint linear in momenta. In this case, the relationship between the constraint functions and the invariance group is more obscure. Indeed, if one computes the Poisson brackets between the constraint functions, one does not find the Lie algebra $\operatorname{diff}(M)$ of the diffeomorphism group, but rather the open algebra of hypersurface deformations (see, e.g., the first reference in Ref. 1). The hypersurfaces being deformed are the Cauchy surfaces on which the canonical variables are defined. Therefore, insofar as the role of the diffeomorphism group is concerned, the canonical treatment of the classical and quantum dynamics of generally covariant systems is quite different from other "manifestly covariant" formulations. It is usually maintained that the loss of covariance is an unavoidable by-product of any canonical formalism. However, as emphasized by Kuchař and Isham, ${ }^{2}$ space-time covariance is not so much lost as it appears in a different guise. This is well illustrated by the canonical formulation of ordinary Minkowski space field theory: There, manifest Poincaré invariance is lost in the usual sense; nevertheless, the phase space does carry a

\footnotetext{
a) Present address: Space Research Institute, Florida Institute of Technology, Melbourne, FL 32901.
}

representation of the Poincaré group via the energy- and angular-momentum tensors. This is, of course, how one knows the canonical formulation is Poincare covariant.

In Ref. 2 it was shown how a similar game can be played with $\operatorname{Diff}(M)$. The key observation made in Ref. 2 is that the needed link between intrinsically space-time quantities, i.e., diffeomorphisms and canonically defined quantities, i.e., constraints, is given by the embeddings that implant the Cauchy surfaces into the space-time. By explicitly incorporating the embeddings into the phase space, one links the action of space-time diffeomorphisms with the corresponding hypersurface deformations. More specifically, given $V \in \operatorname{diff}(M)$, one finds the corresponding phase space function, the "diffeomorphism Hamiltonian" $H(\vec{V})$, by composing a pair of Lie algebra antihomomorphisms. The first antihomomorphism is the familiar map from $\operatorname{diff}(M)$ into the commutator algebra of (complete) vector fields on $M$. The vector field $\vec{V}$ corresponding to $V$ is then restricted to a given embedding and one uses the resulting functional of the embeddings as a smearing field for an appropriate combination of the embedding momenta and the Hamiltonian and momentum constraint functions. This map from the commutator algebra of vector fields into the Poisson bracket algebra of phase space functions is another antihomomorphism. The net effect is a homomorphism from $\operatorname{diff}(M)$ into the phase space Poisson bracket algebra $V \rightarrow \vec{V} \rightarrow H(\vec{V})$. In Ref. 2 this technique was utilized to represent $\operatorname{diff}(M)$ on the phase space of a parametrized scalar field and on an extended phase space for general relatively obtained by using Gaussian coordinate conditions.

In this paper we will investigate the above ideas in the context of the Hamiltonian formulation of a closed bosonic string. Our reason for studying this relatively simple system comes in two parts. First, as is well known, the string provides the most promising (or at least the most popular) framework for unifying all fundamental forces, including 
gravity, in a quantum mechanical setting. One of the central features of string theory is the important role that world sheet symmetries of the first-quantized formalism play in defining the properties of the second-quantized field theoretic formalism. Now, while lip service is usually paid to the world sheet diffeomorphism group, the constraint algebra for the string is actually the Lie algebra conf $(M, g)$ of the group Conf $(M, g)$ of conformal isometries of the metric $g$ on $M$, where $M$ is the manifold which is embedded as a world sheet in Minkowski space. It is this symmetry group that has played the main role in the canonically quantized theory. Thus to date, the role (if any) of the full two-dimensional diffeomorphism group [of which $\operatorname{Conf}(M, g)$ is a subgroup] has remained obscure in the canonical approach to string quantum mechanics and string field theory. From the point of view of the Polyakov formalism, ${ }^{3,4}$ the role of $\operatorname{Diff}(M)$ is more pronounced. For example, the origin of the critical dimension can be seen as a consequence of one's inability to define a functional integration measure which is simultaneously diffeomorphism and Weyl invariant. A direct translation of the results of the Polyakov formalism into the vernacular of the canonically quantized string is not available. By making the canonical role of $\operatorname{Diff}(M)$ explicit, one should, to some degree, be able to unify the canonical and covariant (Polyakov) quantization schemes.

Our second purpose for studying the string de-emphasizes its role as providing a "theory of everything" and instead stresses the role of the string as a simple paradigm for studying the quantization of more conventional theories of gravity. The string, like gravity, is a generally covariant system with Hamiltonian and momentum constraints. Unlike gravity, the string is sufficiently simple such that one can study the canonical quantization process in some detail. By studying the quantum mechanical status of $\operatorname{Diff}(M)$ in this simple setting, we hope to shed light on corresponding issues in quantum gravity. ${ }^{2}$ For example, one can investigate the effect of operator-ordering difficulties and/or anomalies on quantum mechanical covariance within the framework of canonical quantization. A preliminary examination of this particular question is given in Ref. 5 .

This paper is meant to serve primarily as performing the necessary classical groundwork for studying the above quantum mechanical issues. Our goal will be to study several ways in which one can naturally incorporate $\operatorname{diff}(M)$ into the Hamiltonian formulation of the closed string. With proper attention to boundary conditions, the results obtained can be easily translated into the open string framework.

We organize the paper as follows. Section II is mainly devoted to preliminaries and is divided into two parts. Section II A summarizes the Hamiltonian formulation of parametrized harmonic maps from a cylindrical space-time into a (possibly curved) target manifold. The way we represent $\operatorname{diff}(M)$ and conf $(M, g)$ here is to serve as the model for all the work to follow. Section II B contains a sketch of the relevant aspects of the canonical formulation of the closed bosonic string. In Sec. III we utilize the techniques of Ref. 2 to represent $\operatorname{diff}(M)$ on extended phase spaces associated with the conformal and harmonic gauges. In Sec. IV we show that one can go quite a long way toward extracting the embeddings and their conjugate momenta directly from the usual string phase space of Sec. II. In contrast to Sec. III (or Ref. 2), no gauge fixing is needed here, although for technical reasons, it is convenient to extend slightly the conventional string phase space. With an eye on quantization, in Sec. V and we construct BRST (Becchi-Rouet-Stora-Tyutin) invariant extensions of the $\operatorname{diff}(M)$ representatives obtained in Secs. III and IV. In Sec. VI we comment on the distinguished role of the group $\operatorname{Conf}(M, g)$ of conformal isometries as both symmetry and bona fide dynamical groups. We discuss the results obtained and the corresponding implications for their quantum mechanical counterparts in Sec. VII. We have provided three appendices. Appendix A summarizes some rudimentary features of the two-dimensional conformal group. Needed results from the BRST formalism are given in Appendix B. Finally, we summarize our notation and conventions in Appendix $\mathrm{C}$.

\section{PRELIMINARIES}

\section{A. Parametrized scalar fields on the cylinder}

We consider a set of massless scalar fields $\varphi^{A}$ $(A=1, \ldots, d)$ propagating on a globally hyperbolic cylindrical space-time $(M, g)$, where $M=R \times S^{1}$ and $g_{a b}$ is a fixed, externally prescribed metric of Lorentzian signature. The action functional describing the fields is taken to be

$$
S[\varphi]=-\frac{1}{2} \int_{M} \sqrt{-g} g^{a b} G_{A B} \nabla_{a} \varphi^{A} \nabla_{b} \varphi^{B}
$$

In (2.1) we have introduced a "target space" metric $G_{A B}$ which is a nondegenerate ultralocal function of the scalar fields. The stationary points of $S[\varphi]$ correspond to harmonic maps $\varphi^{A}: M \rightarrow M^{d}$, where $M^{d}$ is a $d$-dimensional target space

$$
\begin{aligned}
\frac{\delta S}{\delta \varphi^{A}} & =\sqrt{-g} g^{a b}\left(\nabla_{a}\left(G_{A B} \nabla_{b} \varphi^{B}\right)-\frac{1}{2} G_{B D, A} \nabla_{a} \varphi^{B} \nabla_{b} \varphi^{D}\right) \\
& =0
\end{aligned}
$$

subject to the boundary conditions that the fields are fixed at some initial and final times.

The action can be cast into Hamiltonian form by introducing a foliation

$$
X: R \times S^{1} \rightarrow M .
$$

The foliation is a one-parameter (denoted $\tau$ ) family of embeddings of a circle into $M$. Since the space-time is globally hyberbolic, the map (2.3) will be taken to be a diffeomorphism. In terms of local coordinates $\mathrm{X}^{\alpha}$ and $\sigma \in[0,2 \pi]$ on $M$ and $S^{1}$, respectively, the foliation is described by specifying the coordinate location of the embedded circles:

$$
\mathrm{X}^{\alpha}=X^{\alpha}(\sigma, \tau)
$$

The embedded circles are to serve as Cauchy surfaces for $M$ and hence are spacelike with respect to $g_{a b}$. This means that the metric $\gamma$ induced in each leaf $X_{\tau}$ of the foliation

$$
\gamma=X_{r}^{*} g
$$

or 


$$
\gamma(\sigma):=\gamma_{11}(\sigma)=g_{\alpha \beta}\left(X_{\tau}(\sigma)\right) X^{\alpha}{ }_{, 1} X^{\beta}{ }_{, 1}
$$

is positive definite $\left(X^{\alpha}{ }_{1}=\partial X^{\alpha} / \partial \sigma\right)$. Alternatively, at each embedding there exists a unique timelike normal covector $n_{\alpha}$ defined via

$$
\begin{aligned}
& X_{, 1}^{\alpha} n_{\alpha}=0, \\
& g^{\alpha \beta} n_{\alpha} n_{\beta}=-1,
\end{aligned}
$$

where $n^{\alpha}=g^{\alpha \beta} n_{\beta}$ is future directed. Through (2.6) and (2.7) $n_{\alpha}$ is defined as a fixed functional of the embeddings.

Expression (2.4) can be viewed as providing a coordinate transformation between $\mathrm{X}^{\alpha}$ and $\sigma^{\alpha}=(\tau, \sigma)$. The oneparameter set of embedded circles then arises as the level surfaces $\tau=$ const. Neighboring embeddings, labeled by $\tau$ and $\tau+\delta \tau$, are connected by the deformation vector

$$
N^{\alpha}:=\frac{\partial X^{\alpha}}{\partial \tau}=: \dot{X}^{\alpha}
$$

We decompose $N^{\alpha}$ into its components parallel and perpendicular to the embedding:

$$
N^{\alpha}=N^{1} n^{\alpha}+N^{1} X_{, 1}^{\alpha},
$$

where

$$
\begin{aligned}
& N^{\perp}=-n_{\alpha} N^{\alpha}, \\
& N^{1}=X_{\alpha}^{1} N^{\alpha}, \\
& X_{\alpha}^{1}:=g_{\alpha \beta} \gamma^{11} X_{, 1}^{\beta} .
\end{aligned}
$$

In standard terminology $N^{1}$ is known as the lapse function and $N^{1}$ is known as the shift vector. Any tensor field on $M$ may be restricted to a given embedding and likewise decomposed into tangential and normal pieces. In particular, $\nabla_{a} \varphi^{A}$ and $g_{a b}$ may be decomposed and pulled back to $R \times S^{1}$, yielding the hypersurface form of the action:

$$
\begin{aligned}
S[\varphi]= & \int_{R \times S^{1}} \frac{1}{2} N^{1} \sqrt{\gamma} G_{A B}(\varphi)\left[( N ^ { 1 } ) ^ { - 2 } \left(-\dot{\varphi}^{A}\right.\right. \\
& \left.\left.+\varphi^{A}{ }_{, 1} N^{1}\right)\left(-\dot{\varphi}^{B}+\varphi^{B}{ }_{, 1} N^{1}\right)-\gamma^{11} \varphi^{A}{ }_{, 1} \varphi^{B}{ }_{11}\right] .
\end{aligned}
$$

The momenta $\pi_{A}$ conjugate to $\varphi^{A}$ are easily obtained, from which we can pass to the Hamiltonian form of the action:

$$
S[\varphi, \pi]=\int_{R \times S^{\prime}}\left(\pi_{A} \dot{\varphi}^{A}-N^{\perp} h_{1}-N^{1} h_{1}\right),
$$

where

$$
\begin{aligned}
& h_{\perp}=\gamma^{-1 / 2} h=\gamma^{-1 / 2}\left(\frac{1}{2} G^{A B} \pi_{A} \pi_{B}+\frac{1}{2} G_{A B} \varphi^{A}{ }_{, 1} \varphi^{B}{ }_{, 1}\right), \\
& h_{1}=\pi_{A} \varphi^{A}{ }_{, 1} .
\end{aligned}
$$

The functional $S[\varphi, \pi]$ is to be varied with respect to $\varphi^{A}$ and $\pi_{A}$ with $N^{1}, N^{1}$, and $\gamma$ treated as fixed external functions. We will gain some simplicity in what follows if we rescale the lapse function to be a scalar density of weight minus 1 (equivalent to a vector in this one-dimensional case):

$$
N:=\gamma^{-1 / 2} N^{1} .
$$

The action then takes the form

$$
S[\varphi, \pi]=\int_{R \times S^{\prime}}\left(\pi_{A} \dot{\varphi}^{A}-N h-N^{1} h_{1}\right),
$$

where $h$ is a scalar density of weight 2 . The energy and momentum densities $\left(h, h_{1}\right)$ are independent of the metric induced on the embedding. All foliation and metric dependence is concentrated in $N$ and $N^{1}$.

A significant feature of the lapse density and shift vector is that they are "Weyl invariant," i.e., invariant under conformal rescalings of the metric $g_{a b}$. This can be seen from (2.5), (2.7), (2.11), and (2.16). If we perform a conformal rescaling

$$
\bar{g}_{a b}=e^{2 \omega(X)} g_{a b},
$$

then

$$
\bar{n}_{\alpha}=e^{\omega} n_{\alpha}, \quad \bar{\gamma}_{11}=e^{2 \omega} \gamma_{11} .
$$

Hence

$$
\begin{aligned}
& \bar{N}^{1}=\bar{\gamma}^{11} \bar{g}_{\alpha \beta} X^{\beta}{ }_{1,} N^{\alpha}=N^{1}, \\
& \bar{N}=\bar{\gamma}^{-1 / 2} \bar{N}^{1}=\left[e^{2 \omega} \gamma\right]^{-1 / 2}\left[e^{\omega} N^{1}\right]=N .
\end{aligned}
$$

This, of course, is just a reflection of the fact that only the Weyl invariant combination $\sqrt{-g} g^{a b}$ enters the action (2.1).

\section{The Hamiltonian}

$$
H=\int_{S^{\prime}}\left(N h+N^{1} h_{1}\right)
$$

generates the dynamical flow in the phase space $\Gamma_{0}$, which is the cotangent bundle (with the usual $L^{2}$ dual) over the space of smooth maps from the circle into $M^{d}: \Gamma_{0}=T^{*} C^{\infty}\left(S^{1}, M^{d}\right)$. The variables $\left(\varphi^{A}, \pi_{B}\right)$ constitute a canonical chart on $\Gamma_{0}$, with their only nonvanishing Poisson brackets being

$$
\left[\varphi^{A}(\sigma), \pi_{B}\left(\sigma^{\prime}\right)\right]=\delta_{B}^{A} \delta\left(\sigma, \sigma^{\prime}\right) .
$$

The parametrization process, to be described shortly, allows us to represent the Lie algebra $\operatorname{diff}(M)$ on an extended version of $\Gamma_{0}$. However, first we will indicate why $\Gamma_{0}$ alone cannot carry a natural representation of $\operatorname{diff}(M)$. To do this it is somewhat more efficient to look at the space of solutions $\mathscr{S}$ of (2.2) rather than $\Gamma_{0}$ directly. Since the Cauchy problem for (2.2) is well posed, we know that every point in $\Gamma_{0}$ uniquely determines a point in $\mathscr{S}$.

Given a solution $\varphi^{A}$ to (2.2), the natural action of $\operatorname{Diff}(M)$ is by pullback (this is the right action). Thus if $\phi \in \operatorname{Diff}(M)$, the induced action on $\mathscr{S}$ is given by

$$
\bar{\varphi}^{A}:=\phi^{*} \varphi^{A}=\varphi^{A} \circ \phi .
$$

Near the identity diffeomorphism, $\bar{\varphi}^{A}$ and $\varphi^{A}$ differ by the Lie derivative along the vector field $V^{a}$ which generates $\phi$ :

$$
\delta \varphi^{A}:=\bar{\varphi}^{A}-\varphi^{A}=\mathscr{L}_{\nu} \varphi^{A} .
$$

A straightforward computation shows that $\bar{\varphi}^{A} \in \mathscr{S}$ if and only if

$\nabla_{a}\left(C^{a b}(\vec{V}) G_{A B} \nabla_{b} \varphi^{B}\right)-\frac{1}{2} C^{a b}(\vec{V}) G_{B D, A} \nabla_{a} \varphi^{B} \nabla_{b} \varphi^{D}=0$,

where

$$
C^{a b}(\vec{V}):=\nabla^{a} V^{b}+\nabla^{b} V^{a}-g^{a b} \nabla_{c} V^{c} .
$$

For an arbitrary vector field, (2.22) does not vanish, so that there is no (natural) action of $\operatorname{Diff}(M)$ on $\mathscr{S}$ and hence no action on $\Gamma_{0}$. Note that the vanishing of $C^{a b}$ is the requirement that $V^{a}$ be a conformal Killing vector. Hence we can 
represent on $\mathscr{S}$ the subalgebra conf $(M, g)$ of conformal isometries. This, of course, is just a reflection of the fact that $\operatorname{Conf}(M, g)$ is a symmetry group for this Weyl invariant field theory. Moreover, any solution of the conformal Killing equation is completely determined by its restriction to a Cauchy surface (see Appendix A). We therefore expect to be able to realize conf $(M, g)$ on $\Gamma_{0}$. This can be done as follows.

Let $v^{a}$ denote a conformal Killing vector, thus satisfying

$$
C_{a b}(\vec{v})=0
$$

in the coordinate chart $\mathrm{X}^{\alpha}$ its components are the local functions $v^{\alpha}$ on $M$ :

$$
v^{\alpha}=v^{a}\left(d \mathrm{X}^{\alpha}\right)_{a} .
$$

Using the embedding $X^{\alpha}(\sigma)$ we can pull these two functions back to the circle:

$$
v^{\alpha}(\sigma):=v^{\alpha}(X(\sigma))
$$

It is straightforward to verify that the map from $\operatorname{conf}(M, g)$ into $C^{\infty}\left(\Gamma_{0}, R\right)$ given by

$$
v \rightarrow \vec{v} \rightarrow h(\vec{v}) \text {, }
$$

with

$$
h(\vec{v}):=\int_{S^{\prime}} v^{\alpha}(\sigma) h_{\alpha}
$$

and

$$
h_{\alpha}=-\gamma^{-1 / 2} n_{\alpha} h+X_{\alpha}^{1} h_{1}
$$

is an antihomomorphism, i.e., for two conformal Killing vectors $v^{a}$ and $w^{a}$,

$$
[h(\vec{v}), h(\vec{\omega})]=h([\vec{v}, \vec{\omega}]) .
$$

The reason we obtain an antihomomorphism stems from the following facts: The Lie bracket is minus the conventional commutator of vector fields ${ }^{2}$ and the action of $\operatorname{Conf}(M, g)$ on $\mathscr{S}$ is the right action. The antihomomorphism depends vitally on the way in which $v^{a}$ is locked into the metric and the embedding through (2.23) and (2.24), respectively. Relation (2.27) fails for arbitrary vector fields. That $h(\vec{v})$ does in fact represent a symmetry is verified by computing its dynamical evolution via the Hamiltonian (2.18), from which it is seen that $h(\vec{v})$ is a constant of motion. This result also depends crucially on the fact that $v^{\alpha}(\sigma)$ is the restriction to an embedding of a conformal Killing vector.

The inability to represent more than the subalgebra $\operatorname{conf}(M, g)$ of $\operatorname{diff}(M)$ is easily traced to the fact that we are not letting $\operatorname{Diff}(M)$ act on the space-time metric. In the Hamiltonian framework, $g_{a b}$ is a fixed functional of the embeddings, which are themselves dynamically inert. The parametrization procedure allows $\operatorname{Diff}(M)$ to act on the metric by adjoining the embeddings (and their conjugate momenta) to the phase space and then utilizing the natural left action of $\operatorname{Diff}(M)$ on the embeddings.

Thus let $\phi \in \operatorname{Diff}(M)$ and $X$ be an embedding, i.e.,

$$
\phi: M \rightarrow M, \quad X: S^{1} \rightarrow M .
$$

Then we have

$$
\bar{X}: S^{1} \rightarrow M
$$

via

$$
\bar{X}=\phi \circ X .
$$

Of course, this action of $\operatorname{Diff}(M)$ is not guaranteed to preserve the spacelike character of the embedding. Given an embedding, there will always be some diffeomorphism which yields a nonspacelike embedding. Since we aim to work with a phase space representation of the Cauchy problem, we are obliged to use initial data placed on spacelike surfaces only. Hence we certainly cannot expect to represent the full diffeomorphism group on (the extension of) $\Gamma_{0}$. By the same token, there will always exist an open neighborhood of the identity in $\operatorname{Diff}(M)$ which will map spacelike circles into spacelike circles. We can therefore aspire to represent the Lie algebra $\operatorname{diff}(M)$.

The parametrization process is facilitated by writing the phase space action (2.17) in the form

$$
S[\varphi, \pi]=\int_{R \times S^{\prime}}\left(\pi_{A} \dot{\varphi}^{A}-\dot{X}^{\alpha} h_{\alpha}\right),
$$

where $h_{\alpha}$ is given in (2.26). We now adjoin $X^{\alpha}(\sigma)$ and its conjugate momenta $P_{\alpha}(\sigma)$ to the phase space, so that we work in an extended phase space $\Gamma_{0}^{\prime}$ $=T^{*}\left[C^{\infty}\left(S^{1}, M^{d}\right) \times E m b_{g}\left(S^{1}, M\right)\right]$, where the subscript $g$ indicates that the embeddings are to be spacelike with respect to $g_{a b}$. The only nonvanishing Poisson brackets between $X^{\alpha}(\sigma)$ and $P_{\beta}(\sigma)$ are

$$
\left[X^{\alpha}(\sigma), P_{\beta}\left(\sigma^{\prime}\right)\right]=\delta_{\beta}^{\alpha} \delta\left(\sigma, \sigma^{\prime}\right) .
$$

Since the embedding "velocity" $\dot{X}^{\alpha}$ enters (2.29) linearly, the definition of $P_{\alpha}$ is a constraint:

$$
\mathbf{h}_{\alpha}:=P_{\alpha}+h_{\alpha} \approx 0 .
$$

Next, we adjoin to the action the constraints, smeared with the Lagrange multipliers $N^{\alpha}$, to yield the phase space action on $\Gamma_{0}^{\prime}$ :

$$
S[\varphi, \pi, X, P, \vec{N}]=\int_{R \times S^{\prime}}\left(\pi_{A} \dot{\varphi}^{A}+P_{\alpha} \dot{X}^{\alpha}-N^{\alpha} \mathbf{h}_{\alpha}\right) .
$$

The action is now to be varied freely with respect to all of its arguments. The equations of motion associated with (2.31) for $\left(\varphi^{A}, \pi_{B}\right)$ are the usual ones; they are equivalent to $(2.2)$ : In Hamiltonian form they are

$$
\dot{\varphi}^{A}=\left[\varphi^{A}, \mathbf{h}(\vec{N})\right], \quad \dot{\pi}_{A}=\left[\pi_{A}, \mathbf{h}(\vec{N})\right],
$$

where

$$
\mathbf{h}(\vec{N})=\int_{S^{\prime}} N^{\alpha} \mathbf{h}_{\alpha} .
$$

We also have

$$
\dot{X}^{\alpha}=\left[X^{\alpha}, \mathbf{h}(\vec{N})\right]=N^{\alpha},
$$

which justifies our use of the symbol $N^{\alpha}$ for the multipliers. Finally, the Hamilton equations for $P_{\alpha}$ read as

$$
\dot{P}_{\alpha}=\left[P_{\alpha}, \mathbf{h}(\vec{N})\right]=-\frac{\delta}{\delta X^{\alpha}} \mathbf{h}(\vec{N}) .
$$

The $X$ dependence of $\mathbf{h}(\vec{N})$ comes from the factors $\gamma^{-1 / 2} n_{\alpha}$ and $X_{\alpha}^{1}$ in (2.26). From the constraints (2.30) we see that the embedding momenta $P_{\alpha}$ are combinations of the energy and momentum densities $h$ and $h_{1}$. The equations of motion for $\boldsymbol{P}_{\alpha}$ just amount to the corresponding conservation laws. 
Alternatively, these equations guarantee that the constraints (2.30) are preserved in time; this implies that they are first class in the terminology of Dirac. In fact, it is straightforward to verify that the Poisson algebra of the functions $h(\vec{N})$ is Abelian:

$$
[\mathbf{h}(\vec{N}), \mathbf{h}(\vec{M})]=0
$$

even off the constraint surface (2.30) (by definition, the first class nature of the constraints guarantees $(2.33)$ on the constraint surface).

To recover the constraint functions that generate the non-Abelian algebra of hypersurface deformations, we project the functions $h_{\alpha}$ perpendicular and parallel to a given embedding:

$$
\begin{aligned}
& \mathbf{h}:=\gamma^{1 / 2} n^{\alpha} \mathbf{h}_{\alpha}, \\
& \mathbf{h}_{\mathbf{1}}:=X_{, 1}^{\alpha} \mathbf{h}_{\alpha} .
\end{aligned}
$$

When smeared with a lapse density, $h$ generates the dynamical evolution associated with a normal deformation of the embeddings. Likewise, $h_{1}$ generates the action of $\operatorname{Diff}\left(S^{1}\right)$ on the embeddings and dynamical variables. It will be useful in Sec. IV to have the projected functions written in terms of embeddings registered in a conformal coordinate system. Thus let $T \in(-\infty, \infty)$ and $S \in[0,2 \pi]$ be coordinates on $M$ such that the metric takes the form

$$
g_{T T}=-g_{S S}, \quad g_{S T}=0 .
$$

If we denote $X^{\alpha}(\sigma)=(T(\sigma), S(\sigma))$ and $P_{\alpha}=\left(P_{T}, P_{S}\right)$ we then have

$\mathbf{h}=P_{T} S_{, 1}+P_{S} T_{, 1}+\frac{1}{2}\left(G^{A B} \pi_{A} \pi_{B}+G_{A B} \varphi^{A}{ }_{, 1} \varphi^{B}{ }_{1,1}\right)$,

$\mathbf{h}_{1}=P_{T} T_{, 1}+P_{S} S_{, 1}+\pi_{A} \varphi^{A}{ }_{11}$.

Relation (2.33) allows us to implement $\operatorname{diff}(M)$ on $\Gamma_{0}^{\prime}$ in an analogous fashion to our previous representation of $\operatorname{conf}(M, g)$. Given any (complete) vector field $\vec{U}$ on $M$, we again form two scalars via the coordinate one-form basis. We then pull these functions back along the embedding and use the resulting functions on $S^{1}$ to smear $\mathbf{h}_{\alpha}$ :

$$
\mathbf{h}(\vec{U})=\int_{S^{\prime}} U^{\alpha}(X(\sigma)) \mathbf{h}_{\alpha} .
$$

Using (2.33) it follows immediately that the map from $\operatorname{diff}(M)$ into $C^{\infty}\left(\Gamma_{0}^{\prime}, R\right)$ given by

$$
U \rightarrow \vec{U} \rightarrow \mathbf{h}(\vec{U})
$$

is a homomorphism. [Here we obtain a homomorphism due to the fact that the action of $\operatorname{Diff}(M)$ on the embeddings is the left action.]

$$
[\mathbf{h}(\vec{U}), \mathbf{h}(\vec{V})]=\mathbf{h}(-[\vec{U}, \vec{V}]) .
$$

Physically, we can think of the action of $\operatorname{diff}(M)$ on $\Gamma_{0}^{\prime}$ as follows. Decompose $h(\vec{V})$ as

$$
\mathbf{h}(\vec{V})=\int_{S^{\prime}} V^{\alpha}(\sigma) P_{\alpha}+\int_{S^{\prime}} V^{\alpha}(\sigma) h_{\alpha}:=P(\vec{V})+h(\vec{V}) .
$$

The function $P(\vec{V})$ acts on $T^{*} E m b_{g}\left(S^{1}, M\right)$ with the effect of displacing the embedded circle along the deformation vector $\vec{V}$ by the infinitesimal version of (2.28). The function $h(\vec{V})$ then evolves the canonical data in the correct dynami- cal fashion associated with the Hamiltonian equations. Proceeding along the integral curves of $\vec{V}$, one builds a solution to the Hamiltonian equations with lapse and shift defined by the projections of $\vec{V}$.

\section{B. The closed string}

The action functional for the massless relativistic string can be obtained from (2.1) by taking the target space for the harmonic maps to be $d$-dimensional Minkowski space. Conventionally, one uses an inertial coordinate system $x^{\mu}$, $\mu=0, \ldots, d-1$ on $M^{d}$ in the role of $\varphi^{A}$ to yield

$$
S[g, x]=-\frac{1}{2} \int_{M} \sqrt{-g} g^{a b} \eta_{\mu \nu} \nabla_{a} x^{\mu} \nabla_{b} x^{\nu}
$$

Aside from the change in notation, the primary difference between the variational principles associated with (2.1) and (2.40) is that in the former we extremize the action with the metric treated as given, i.e., fixed. In the variational principle for the string we vary both $x^{\mu}$ and $g_{a b}$. As a result, when we extremize (2.40) we obtain two sets of equations:

$$
\frac{\delta S}{\delta x^{\mu}}=\sqrt{-g} g^{a b} \eta_{\mu \nu} \nabla_{a} \nabla_{b} x^{\nu}=0
$$

and

$\frac{2}{\sqrt{-\mathrm{g}}} \frac{\delta S}{\delta g_{a b}}=\eta_{\mu \nu}\left(\nabla^{a} x^{\mu} \nabla^{b} x^{\nu}-\frac{1}{2} g^{a b} g^{c d} \nabla_{c} x^{\mu} \nabla_{d} x^{\nu}\right)=0$.

As before, (2.41) is the equation for a harmonic map $x: M \rightarrow M^{d}$. However, the metric $g_{a b}$ is now coupled to the induced metric on the world sheet through (2.42). Specifically, (2.42) tells us that the Weyl invariant parts of the two metrics are equal:

$$
g_{a b}=e^{2 \omega}\left(x^{*} \eta\right)_{a b} .
$$

Here $\omega$ is a function on $M$ which cannot be determined, owing to the Weyl invariance of the theory. In contrast to the previous situation, the system of equations (2.41) and (2.42) is degenerate in the sense that the equations obey an infinite set of ("Bianchi") identities

$$
\int_{M}\left(\frac{\delta S}{\delta x^{\mu}} \mathscr{L}_{\nu} x^{\mu}+\frac{\delta S}{\delta g_{a b}} \mathscr{L}_{\nu} g_{a b}\right)=0,
$$

where $V^{a}$ is any vector field on $M$ with compact support. The identities (2.43) are a direct consequence of the invariance of the action with respect to variations of $\left(x^{\mu}, g_{a b}\right)$ induced by diffeomorphic mappings of $M$ onto itself. As a direct consequence of the diffeomorphism invariance of the action we have the following immediate result: If $(x, g)$ is a solution to the equations of motion, then for $\phi \in \operatorname{Diff}(M),\left(\phi^{*} x, \phi^{*} g\right)$ is also a solution. The identities (2.43), while complicating the Cauchy problem for (2.41) and (2.42), thus serve to indicate that the space of solutions to the equations of motion admits an action of $\operatorname{Diff}(M)$. From our earlier discussion we also know that $\operatorname{Conf}(M, g)$ is a symmetry group for the system: Given a solution $(x, g)$ and $\psi \in \operatorname{Conf}(M, g)$, both $\left(\psi^{*} x, g\right)$ and $\left(x, \psi^{*} g\right)$ will be solutions [of course, we can also let $\psi$ act on the string and metric variables simultaneously, but this is just a special case of the $\operatorname{Diff}(M)$ action ]. 
The Hamiltonian formulation for the string is obtained in exactly the same fashion as for the harmonic maps of Sec. II A. Again, the important new feature that enters is that the metric is now freely variable. The phase space $\Gamma$ can be taken to be the cotangent bundle over the space of smooth embeddings of a circle into $M^{d}$ which are spacelike with respect to the target space metric $\eta_{\mu \nu}$ :

$$
\Gamma=T^{*}\left[E m b_{\eta}\left(S^{1}, M^{d}\right)\right] .
$$

A canonical coordinate patch is given by the pair $\left(x^{\mu}{ }_{s} p_{v}\right)$ :

$$
\left[x^{\mu}(\sigma), p_{\nu}\left(\sigma^{\prime}\right)\right]=\delta_{\nu}^{\mu} \delta\left(\sigma, \sigma^{\prime}\right) \text {. }
$$

The action functional on $R \times S^{1}$ takes the form

$$
S[x, p, \vec{N}]=\int_{R \times S^{\prime}}\left(p_{\mu} \dot{x}^{\mu}-N \mathscr{H}-N^{\prime} \mathscr{H}_{1}\right),
$$

where

$$
\mathscr{H}=\frac{1}{2}\left(\eta^{\mu v} p_{\mu} p_{v}+\eta_{\mu v} x^{\mu}{ }_{, 1} x^{v}{ }_{1}\right)
$$

and

$$
\mathscr{H}_{1}=p_{\mu} x^{\mu}{ }_{1} .
$$

From the Hamiltonian point of view, the distinction between (2.13) and (2.44) is that in the former case the lapse and shift are fixed, externally prescribed functions on $S^{1}$, while in the present case they are independently variable: Thus they serve as Lagrange multipliers enforcing the constants

$$
\begin{aligned}
& \mathscr{H} \approx 0, \\
& \mathscr{H}_{1} \approx 0 .
\end{aligned}
$$

Note that the constraints (2.47) and (2.48) arise in an entirely different manner than those for the parametrized scalar fields: There, the constraints appear after adjoining a nondynamical set of variables (the embeddings and their conjugate momenta) to the phase space. Here, no adjoining is necessary and, as one often says, the theory is "already parametrized."

The Hamiltonian

$$
H(\vec{N}):=\int_{S^{\prime}}\left(N \mathscr{H}+N^{1} \mathscr{H}_{1}\right)
$$

generates the dynamical flow, which depends on a choice for the lapse and shift:

$$
\begin{aligned}
& \dot{x}^{\mu}=\left[x^{\mu}, H(\vec{N})\right]=N p^{\mu}+N^{1} x^{\mu}{ }_{, 1}, \\
& \dot{p}_{\mu}=\left[p_{\mu}, H(\vec{N})\right]=\partial_{1}\left(N x_{\mu, 1}+N^{1} p_{\mu}\right) .
\end{aligned}
$$

From (2.50) we see that the super-Hamiltonian

$$
H(N)=\int_{S^{\prime}} N \mathscr{H}
$$

generates the dynamical evolution associated with a normal deformation of the embeddings. The supermomentum

$$
H\left(N^{1}\right)=\int_{S^{\prime}} N^{1} \mathscr{H}_{1}
$$

generates the motion in $\Gamma$ associated with a tangential deformation of the circles. This, of course, is just an action of $\operatorname{Diff}\left(S^{1}\right)$ on the phase space which is generated by the vector $N^{1}$.
The constraints (2.47) and (2.48) are complete in the sense that if they are satisfied on some initial embedding, i.e., at some initial $\tau$, then they are satisfied everywhere along the dynamical flow (2.50) and (2.51). This is guaranteed by the "Dirac algebra" which the constraint functions satisfy:

$$
\begin{aligned}
& {[H(N), H(M)]=H\left(L^{1}\right),} \\
& {\left[H(N), H\left(M^{1}\right)\right]=H(K),} \\
& {\left[H\left(N^{1}\right), H\left(M^{1}\right)\right]=H\left(J^{1}\right),}
\end{aligned}
$$

where

$L^{1}=N \partial_{1} M-M \partial_{1} N, \quad K=N \partial_{1} M^{1}-M^{1} \partial_{1} N$, $J^{1}=N^{1} \partial_{1} M^{1}-M^{1} \partial_{1} N^{1}$.

In contrast to general relativity or parametrized theories on backgrounds of dimension greater than 2, the Dirac algebra in two dimensions is a Lie algebra. This algebra is in fact isomorphic to $\operatorname{diff}\left(S^{1}\right) \oplus \operatorname{diff}\left(S^{1}\right)$ [which in turn is isomorphic to conf $(M, g)$; see Appendix A], as can be seen by rearranging the constraints as follows:

$$
\left( \pm \mathscr{H}:=\frac{1}{2}\left(\mathscr{H} \pm \mathscr{H}_{1}\right) \approx 0\right. \text {. }
$$

When smeared with vector fields on $S^{1}$, the constraint algebra becomes

$$
\begin{aligned}
& {\left[{ }_{( \pm)} H\left(N^{1}\right),_{( \pm)} H\left(M^{1}\right)\right]= \pm{ }_{( \pm)} H\left(\mathscr{L}_{N} M^{1}\right),} \\
& {\left[{ }_{(+)} H\left(N^{1}\right),{ }_{(-)} H\left(M^{1}\right)\right]=0,}
\end{aligned}
$$

which is $\operatorname{diff}\left(S^{1}\right) \oplus \operatorname{diff}\left(S^{1}\right)$. Note that the equivalence between vectors and scalar densities of weight minus 1 , which occurs only in one dimension, is crucial for the existence of the algebraic structure just described.

We are now in a position to elaborate on the work to follow. We are aspiring to find a homomorphic mapping from $\operatorname{diff}(M)$ into the Poisson algebra of functions $C^{\infty}(\Gamma, R)$. Obviously, the action of $\operatorname{Diff}(M)$ on $\Gamma$ should lead to the appropriate dynamical evolution as we move from point to point in $M$. One therefore expects that the natural place to look for such a realization of $\operatorname{diff}(M)$ is the constraint functions (2.52) and (2.53). Indeed, as we have seen, these functions do form a Lie algebra. There are problems, however. First, the algebra is the wrong algebra $\left[\operatorname{diff}\left(S^{1}\right) \oplus \operatorname{diff}\left(S^{1}\right)\right.$ rather than $\left.\operatorname{diff}(M)\right]$. More important, however, is the fact that there is no (obvious) way to link phase space quantities, defined on $S^{1}$, to space-time quantities, in particular vector fields on $M$. The homomorphism obtained via the parametrization procedure of Sec. II A relied heavily on the fact that the metric on $M$ was known in advance. This allowed for the canonical-covariant connection to be obtained since the metric (used to define projections) was a fixed, known functional of the embeddings. In the present situation, the metric is effectively a part of the configuration space (albeit a nondynamical part) to be varied independent of the embedding. The metric and foliation are now decoupled and in fact, the embeddings no longer directly feature in the formalism. Consequently, a direct mimicry of the procedure used in Sec. II A is impossible. Based on these considerations, two courses of action suggest themselves. One can try to reestablish the coupling between the metric and embedding by a form of "gauge fixing." This 
line of attack, originally devised for geometrodynamics by Isham and Kuchar̆, ${ }^{2}$ is the subject of Sec. III. Alternatively, one can try to take advantage of the notion that the string is in some sense "already parametrized." In Sec. IV we investigate the degree to which one can extract the embedding variables (and their conjugate momenta) directly from the string phase space $\Gamma$. Success in either of these approaches leads to the desired representation of $\operatorname{diff}(M)$ through the techniques of Sec. II A.

Finally, we point out (with some irony intended) that the same difficulties arise in trying to homomorphically map $\operatorname{conf}(M, g)$ into the Poisson algebra of functions on $\Gamma$. Granted, the phase space does carry the Lie algebra $\operatorname{diff}\left(S^{1}\right) \oplus \operatorname{diff}\left(S^{1}\right)$, which is isomorphic to $\operatorname{conf}(M, g)$, as seen in (2.58). However, the lack of any connection between phase space functions and space-time (conformal Killing) vectors obstructs any attempt at finding a homomorphic mapping. Nevertheless, the fact that the two-dimensional algebra of hypersurface deformations is isomorphic to $\operatorname{conf}(M, g)$ does suggest a fundamental dynamical role for the conformal group. We will elucidate this point in Sec. VI.

\section{REPRESENTATION OF diff(M) ON EXTENDED PHASE SPACES}

Here we shall follow the strategy of Ref. 2 . The basic idea is to recouple the metric on $M$ to the embeddings through gauge-fixing conditions. For aesthetic reasons, we will use an "auxiliary structure" to fix the gauge, so that we always deal with geometrically well-defined objects. We will, however, occasionally pause to make contact with the possibly more familiar coordinate-dependent expressions.

The auxiliary structure that we will need is simply a fixed foliation

$$
\widetilde{Y}: R \times S^{1} \rightarrow M
$$

(which will be a diffeomorphism) and its inverse, denoted $Y$ :

$$
Y:=\widetilde{Y}^{-1}, \quad Y: M \rightarrow R \times S^{1} .
$$

If we fix once and for all local coordinates $\sigma^{(\alpha)}$ on $R \times S^{1}$,

$$
\sigma^{(\alpha)}: R \times S^{1} \rightarrow R^{2},
$$

we can compose them with the map $Y$ to obtain local coordinates $Y^{(\alpha)}$ on $M$ :

$$
Y^{(\alpha)}=\sigma^{(\alpha)} \circ Y, \quad Y^{(\alpha)}: M \rightarrow R^{2} .
$$

While a choice of $\tilde{Y}$ is necessarily ad hoc (we have an infinity of possibilities), we are to think of having made a definite choice. The gauge-fixing procedure will amount to fixing two components of $g_{a b}$ with respect to the given auxiliary structure. This structure will then serve to provide the needed link between the metric and the embeddings which we introduce to construct the Hamiltonian formulation. At any point along the way, we can put a given expression into a more conventional form by choosing coordinates $\mathrm{X}^{\alpha}$ on $M$, which are adapted to the foliation $\widetilde{Y}$ via

$$
\mathrm{X}^{a}=Y^{(\alpha)} .
$$

\section{A. Conformal gauge}

The use of the conformal gauge is ubiquitous in string theory. Among its virtues we have the simplicity of the resulting dynamical equations and the fact that one can always find conformal coordinates which cover all of $M$ except for a set of measure zero.

In terms of the auxiliary structure $Y$, the conformal gauge conditions take the form

$$
\begin{aligned}
& g^{a b} \delta_{(\alpha)(\beta)} \nabla_{a} Y^{(\alpha)} \nabla_{b} Y^{(\beta)}=0, \\
& g^{a b} e_{(\alpha)(\beta)} \nabla_{a} Y^{(\alpha)} \nabla_{b} Y^{(\beta)}=0,
\end{aligned}
$$

where

$$
e_{(\alpha)(\beta)}=\left(\begin{array}{ll}
1 & 1 \\
0 & 0
\end{array}\right)
$$

If we denote by $\widetilde{\gamma}$ the metric induced on the leaves of the foliation,

$$
\widetilde{\gamma}=\widetilde{Y} * g
$$

then the metric can be expressed as a functional of $\tilde{\gamma}$ and $Y$ via

$$
g_{a b}=\tilde{\gamma} \eta_{(\alpha)(\beta)} \nabla_{a} Y^{(\alpha)} \nabla_{b} Y^{(\beta)} .
$$

As summarized in Appendix A, every metric on $M$ is conformal to a flat metric. For an arbitrary auxiliary structure, (3.3) just serves to capture that fact, i.e., (3.3) is simply an invariant parametrization of the space of metrics. By fixing the auxiliary structure we are fixing the flat metric to which $g_{a b}$ is conformally related. The only remaining "degree of freedom" is the conformal factor $\widetilde{\gamma}$. Weyl invariance for the classical string will guarantee that this remaining metric degree of freedom does not play a role in the theory.

If we choose coordinates adapted to the foliation as discussed above, the gauge-fixing conditions (3.1) and (3.2) take the familiar form

$$
g^{00}=-g^{11}, \quad g^{01}=0 .
$$

Alternatively, (3.3) reads as

$$
g_{\alpha \beta}=\tilde{\gamma} \eta_{(\alpha)(\beta)}
$$

in the adapted coordinates $\mathrm{X}^{\alpha}=Y^{(\alpha)}$.

We can now substitute the metric (3.3) into the string action (2.40). The conformal degree of freedom $\tilde{\gamma}$ drops out of the action. This leaves an action that is a functional of the fixed auxiliary structure and the string variables. Only the latter quantities are to be varied; Eqs. (2.47) and (2.48) are now suspended and we have arrived back at the formalism for the harmonic maps of Sec. II A.

Following the Hamiltonian treatment of the parametrized harmonic maps, we now work in an extended phase space $\Gamma^{\prime}$ for the string, which is given by

$$
\Gamma^{\prime}=T^{*}\left[E m b_{\eta}\left(S^{1}, M^{d}\right) \times E m b_{g}\left(S^{1}, M\right)\right] .
$$

The corresponding action functional becomes

$$
S[x, p, X, P, \vec{N}]=\int_{R \times S^{\prime}}\left(p_{\mu} \dot{x}^{\mu}+P_{\alpha} \dot{X}^{\alpha}-N^{\alpha} \mathbf{H}_{\alpha}^{\prime}\right),
$$

where

$$
\mathbf{H}_{\alpha}^{\prime}:=P_{\alpha}+\mathscr{H}_{\alpha} \approx 0
$$


and

$$
\mathscr{H}_{\alpha}=-\gamma^{-1 / 2} n_{\alpha} \mathscr{H}+X_{\alpha}^{1} \mathscr{H}_{1},
$$

with $\mathscr{H}$ and $\mathscr{H}_{1}$ given by (2.45) and (2.46). In (3.6) we have defined $\gamma=X^{*} g$, with $g_{a b}$ given by (3.3). Note that the induced metric ( $\gamma$ or $\tilde{\gamma}$ ) dutifully drops out of the functions (3.6), i.e.,

$$
\mathscr{H}_{\alpha}=\mathscr{H}_{\alpha}[x, p, X] .
$$

This can be verified using the definition of $n_{\alpha}$ [Eqs. (2.6) and (2.7)] and $X_{\alpha}^{1}$ [Eq. (2.11b)] in conjunction with the gauge-fixed metric (3.3). We therefore need not include $\gamma$ (or $\widetilde{\gamma}$ ) in the phase space. Alternatively, the equation of motion for $\gamma$,

$$
\frac{\delta S}{\delta \gamma}=0
$$

is identically satisfied.

The constraints (3.5) obey an Abelian Poisson algebra; consequently, we can immediately construct the desired Lie algebra homomorphism. For $V, W \in \operatorname{diff}(M)$, the map

$$
V \rightarrow \vec{V} \rightarrow \mathbf{H}^{\prime}(\vec{V}),
$$

where

$$
\mathbf{H}^{\prime}(\vec{V})=\int_{S^{\prime}} V^{\alpha}(X(\sigma)) \mathbf{H}_{\alpha}^{\prime},
$$

yields

$$
\left[\mathbf{H}^{\prime}(\vec{V}), \mathbf{H}^{\prime}(\vec{W})\right]=\mathbf{H}^{\prime}(-[\vec{V}, \vec{W}]) .
$$

The symmetry algebra conf $(M, g)$ can also be represented: To represent it as a subalgebra of $\operatorname{diff}(M)$ we use

$$
v \rightarrow \vec{v} \rightarrow \mathbf{H}^{\prime}(\vec{v}),
$$

where $\vec{v}$ is a conformal Killing vector for the metric (3.3). Note that such vectors are in fact independent of $\widetilde{\gamma}$. This representation emphasizes the role of $\operatorname{conf}(M, g)$ as a $d y$ namical subalgebra of $\operatorname{diff}(M)$. As discussed earlier, $\operatorname{Conf}(M, g)$ also acts as a symmetry group on the space of solutions to the string dynamical equations. In Hamiltonian language, this representation is implemented via the following two mappings:

$$
v \rightarrow \vec{v} \rightarrow P(\vec{v}), \quad P(\vec{v})=\int_{S^{\prime}} v^{\alpha} P_{\alpha},
$$

and

$$
v \rightarrow \vec{v} \rightarrow \mathscr{H}(\vec{v}), \quad \mathscr{H}(\vec{v})=\int_{S^{\prime}} v^{\alpha} \mathscr{H}_{\alpha} .
$$

The map (3.9) is a homomorphism from conf $(M, g)$ into the Poisson algebra of functions on $\Gamma^{\prime}$ :

$$
[P(\vec{v}), P(\vec{w})]=P(-[\vec{v}, \vec{w}]),
$$

while (3.10) is an antihomomorphism

$$
[\mathscr{H}(\vec{v}), \mathscr{H}(\vec{w})]=\mathscr{H}([\vec{v}, \vec{w}]) .
$$

As in Sec. II A, the functions $\mathscr{H}(\vec{w})$ are indeed symmetry generators since they are constants of motion. Thus for any vector $\vec{V}$ and any conformal Killing vector $\vec{w}$,

$$
\left[\mathscr{H}(\vec{w}), \mathbf{H}^{\prime}(\vec{V})\right]=0 .
$$

The functions $P(\vec{w})$ are generators of a conditional symmetry, i.e., they are conserved modulo the constraints (3.5):

$$
\left[P(\vec{w}), \mathbf{H}^{\prime}(\vec{V})\right]=\mathbf{H}^{\prime}(-[\vec{w}, \vec{V}]) \approx 0
$$

In exactly the same manner as for the parametrized fields, the functions $H^{\prime}(\vec{V})$ act on $\Gamma^{\prime}$ by displacing the embedding along the deformation vector $\vec{V}$ which generates the diffeomorphism, while simultaneously evolving the string variables along the flow lines of $\vec{V}$. It is important to realize that while we use a conformal coordinate system (in the guise of the auxiliary structure) to describe the kinematics of the dynamical evolution, we are not evolving with some fixed (conformal) foliation. In other words, we still evolve from one arbitrary Cauchy surface to another. The choice of deformation vector chooses the slicing. Having chosen a deformation vector we can, if we like, use the gauge-fixed metric to extract the corresponding lapse and shift. The string evolution is then governed by the following:

$$
\begin{aligned}
& \dot{x}^{\mu}=\left[x^{\mu}, \mathbf{H}^{\prime}(\vec{N})\right]=N p^{\mu}+N^{1} x^{\mu}{ }_{, 1}, \\
& \dot{p}^{\mu}=\left[p^{\mu}, \mathbf{H}^{\prime}(\vec{N})\right]=\partial_{1}\left(N x_{\mu, 1}+N^{1} p_{\mu}\right) .
\end{aligned}
$$

Despite the fact that $\left(N, N^{1}\right)$ obtained from $\vec{V}$ are functionals of the embeddings, Eqs. (3.11) and (3.12) are identical to the usual string equations of motion (2.50), (2.51); the evolution within the extended phase space is still the correct one. This occurs because the induced metric $\gamma$ does not play a role in the formalism. Moreover, even if the metric on $M$ were defined to be the metric induced by virtue of the string embeddings $x^{\mu}$, thus rendering $\gamma$ a functional of the string variables, Weyl invariance would again guarantee that the equations of motion would be correct. (This should be contrasted with the case of geometrodynamics, ${ }^{2}$ where the metric is dynamical and the correct equations of motion are obtained only weakly, i.e., upon imposing the original Hamiltonian and momentum constraints.) On the other hand, the solutions to (3.11) and (3.12) are not in general solutions to the full set of string equations (2.41) and (2.42). To obtain the physical string solutions we must restrict the admissible initial data in the string sector of $\Gamma^{\prime}$ to satisfy the original constraints (2.47) and (2.48). Thus the physically relevent dynamical evolution begins in the subspace $\bar{\Gamma}^{\prime}$ of $\Gamma^{\prime}$ specified by (2.47), (2.48), and (3.5). If initial data are chosen from this subspace, it can be verified that the dynamical evolution remains in $\bar{\Gamma}^{\prime}$. The verification amounts to checking that the complete set of constraint functions $\left(\mathscr{H}, \mathscr{H}_{1}, \mathbf{H}_{\alpha}^{\prime}\right)$ is first class. We have already proven a large part of this in Eqs. (2.54)-(2.56) and (3.8). It only remains to be checked that the original string constraints have weakly vanishing Poisson brackets with the Hamiltonian $\mathbf{H}^{\prime}(\vec{N})$. Conservation of the Hamiltonian constraint is guaranteed since

$$
\begin{aligned}
& {[\mathscr{H}(\sigma), \mathbf{H}(\vec{N})]} \\
& \quad=\left[\mathscr{H}(\sigma), \int_{S^{\prime}} N^{\alpha}\left(-\gamma^{-1 / 2} n_{\alpha} \mathscr{H}+X_{\alpha}^{1} \mathscr{H}_{1}\right)\right] .
\end{aligned}
$$

This expression weakly vanishes because the string constraints obey the Dirac algebra, in particular (2.54) and (2.55) [see, also (5.3)]. Similarly,

$$
\begin{aligned}
& {\left[\mathscr{H}_{1}(\sigma), \mathbf{H}^{\prime}(\vec{N})\right]} \\
& \quad=\left[\mathscr{H}_{1}(\sigma), \int_{S^{\prime}} N^{\alpha}\left(-\gamma^{-1 / 2} n_{\alpha} \mathscr{H}+X_{\alpha}^{1} \mathscr{H}_{1}\right)\right],
\end{aligned}
$$


which weakly vanishes as a result of (2.55) and (2.56) [see, also (5.4) ].

To summarize, we have represented the Lie algebra $\operatorname{diff}(M)$ at the expense of working in an extended phase space $\Gamma^{\prime}$. The representatives $\mathbf{H}^{\prime}(\vec{V})$ of the vector fields $\vec{V}$ on $M$ correctly evolve the initial data. To obtain physical string dynamics, we must restrict the admissible initial data to lie in the subspace $\bar{\Gamma}^{\prime} \subset \Gamma^{\prime}$. If the dynamical evolution begins in $\bar{\Gamma}^{\prime}$, it will stay there.

As mentioned earlier, one of the primary advantages of the conformal gauge lies in the ease with which one can solve the equations of motion. Let us conclude our discussion of the conformal gauge by outlining how this occurs in the Hamiltonian formulation based on the extended phase space $\Gamma^{\prime}$.

We begin by observing that the evolution equations for the string are equivalent to a system of functional differential equations. ${ }^{6}$ To see this, note that any function on the phase space $F \in C^{\infty}\left(\Gamma^{\prime}, R\right)$ is dynamically evolved according to

$$
\dot{F}=\left[F, \mathbf{H}^{\prime}(\vec{N})\right]
$$

The dependence of the evolution on the choice of foliation resides in the deformation vector $N^{\alpha}$, which is freely specifiable. Both $\mathbf{H}^{\prime}(\vec{N})$ and $\dot{F}$ are linear functionals of $N^{\alpha}$ :

$$
\begin{aligned}
& \mathbf{H}^{\prime}(\vec{N})=\int_{S^{\prime}} N^{\alpha} \mathbf{H}_{\alpha}^{\prime}, \\
& \dot{F}=\int_{S^{\prime}} \dot{X}^{\alpha} \frac{\delta F}{\delta X^{\alpha}}=\int_{S^{\prime}} N^{\alpha} \frac{\delta F}{\delta X^{\alpha}} .
\end{aligned}
$$

Using the fact that $N^{\alpha}$ is arbitrary, an equation equivalent to (3.13) is given by

$$
\frac{\delta F}{\delta X^{\alpha}(\sigma)}=\left[F, \mathbf{H}_{\alpha}^{\prime}(\sigma)\right] \text {. }
$$

In particular, we can let $F$ be one of the canonical coordinates on $\Gamma^{\prime}$, thereby establishing a set of functional differential, "many-fingered time" Hamiltonian equations.

We know that the equations of motion will be simple in conformal coordinates, so we choose our coordinates $\mathrm{X}^{\alpha}$ on $M$ to be adapted to the conformal foliation. In particular, define

$\mathrm{X}^{0}=Y^{(0)}=: \mathrm{T}, \quad \mathrm{X}^{1}=Y^{(1)}=: \mathrm{S}, \quad \mathrm{X}^{ \pm}:=\mathrm{T} \pm \mathrm{S}$.

Here, $T$ is a coordinate on $R^{1}, S$ is a coordinate on $S^{1}$, and $\mathrm{X}^{ \pm}$are the associated null coordinates with respect to the metric (3.3). In terms of the latter set of coordinates, the gauge-fixed metric has components given by

$$
g_{++}=0=g_{--}, \quad g_{+-}=-\frac{1}{2} \widetilde{\gamma} .
$$

Given an embedding $X^{\alpha}(\sigma)$, the metric $\gamma$ induced on it is

$$
\gamma_{11}(\sigma)=-\widetilde{\gamma}(\sigma) X^{+}{ }_{, 1} X^{-}{ }_{, 1} .
$$

The embedding is to be spacelike, which implies

$$
X^{+}{ }_{, 1} X^{-}{ }_{, 1}<0 \text {. }
$$

The unit normal covector to the embedding has components in the null coordinate basis given by

$$
n_{ \pm}= \pm \frac{1}{2} \widetilde{\gamma}^{1 / 2}\left(-X^{+}{ }_{, 1} X^{-}{ }_{, 1}\right)^{-1 / 2} X^{\mp}{ }_{, 1} .
$$

Finally, the covector $X_{\alpha}^{1}$ (on $M$ ), defined in (2.11b), has the components

$$
X_{ \pm}^{1}=\frac{1}{2}\left(X^{ \pm}{ }_{, 1}\right)^{-1} \text {. }
$$

Assembling the expressions (3.15)-(3.19) together, the many-fingered time Hamiltonian has components given by

$$
\begin{aligned}
& \mathbf{H}_{+}^{\prime}=P_{+}+\left(X^{+}, 1\right)^{-1} \tilde{\alpha}_{\mu} \tilde{\alpha}^{\mu}, \\
& \mathbf{H}_{-}^{\prime}=P_{-}-\left(X^{-}, 1\right)^{-1} \alpha_{\mu} \alpha^{\mu},
\end{aligned}
$$

where we have introduced the usual notation

$$
\begin{aligned}
& \tilde{\alpha}^{\mu}:=\frac{1}{2}\left(p^{\mu}+x^{\mu}{ }_{1}\right), \\
& \alpha^{\mu}:=\frac{1}{2}\left(p^{\mu}-x^{\mu}{ }_{11}\right) .
\end{aligned}
$$

Note that $\alpha^{\mu}$ and $\tilde{\alpha}^{\mu}$ are scalar densities of weight 1 (covectors) on $S^{1}$.

By substituting each of the canonical variables into the functional Hamiltonian equation (3.14), we obtain

$\frac{\delta P_{+}(\sigma)}{\delta X^{+}\left(\sigma^{\prime}\right)}=\left(X^{+}{ }_{, 1}\left(\sigma^{\prime}\right)\right)^{-2} \tilde{\alpha}_{\mu}\left(\sigma^{\prime}\right) \tilde{\alpha}^{\mu}\left(\sigma^{\prime}\right) \partial_{\sigma} \delta\left(\sigma, \sigma^{\prime}\right)$,
$\frac{\delta P_{-}(\sigma)}{\delta X^{-}\left(\sigma^{\prime}\right)}=-\left(X_{, 1}^{-}\left(\sigma^{\prime}\right)\right)^{-2} \alpha_{\mu}\left(\sigma^{\prime}\right) \alpha^{\mu}\left(\sigma^{\prime}\right) \partial_{\sigma} \delta\left(\sigma, \sigma^{\prime}\right)$,

$\frac{\delta P_{ \pm}(\sigma)}{\delta X^{\mp}\left(\sigma^{\prime}\right)}=0$,

$\frac{\delta X^{\alpha}(\sigma)}{\delta X^{\beta}\left(\sigma^{\prime}\right)}=\delta_{\beta}^{\alpha} \delta\left(\sigma, \sigma^{\prime}\right)$,

$\frac{\delta x^{\mu}(\sigma)}{\delta X^{+}\left(\sigma^{\prime}\right)}=\left(X^{+, 1}\right)^{-1} \tilde{\alpha}^{\mu} \delta\left(\sigma, \sigma^{\prime}\right)$,

$\frac{\delta x^{\mu}(\sigma)}{\delta X^{-}\left(\sigma^{\prime}\right)}=-\left(X^{-}, 1\right)^{-1} \alpha^{\mu} \delta\left(\sigma, \sigma^{\prime}\right)$,

$\frac{\delta p_{\mu}(\sigma)}{\delta X^{+}\left(\sigma^{\prime}\right)}=\left(X^{+}{ }_{, 1}\left(\sigma^{\prime}\right)\right)^{-1} \tilde{\alpha}^{\mu}\left(\sigma^{\prime}\right) \partial_{\sigma} \delta\left(\sigma, \sigma^{\prime}\right)$,

$\frac{\delta p_{\mu}(\sigma)}{\delta X^{-}\left(\sigma^{\prime}\right)}=\left(X^{-}{ }_{.1}\left(\sigma^{\prime}\right)\right)^{-1} \alpha_{\mu}\left(\sigma^{\prime}\right) \partial_{\sigma} \delta\left(\sigma, \sigma^{\prime}\right)$.

Equations (3.23)-(3.30) are to be solved so that they match an initial data set $\left(\bar{X}^{\alpha}, \bar{P}_{\alpha}, \bar{x}^{\mu}, \bar{p}_{\mu}\right)$. The initial data in turn must satisfy the constraints (2.47), (2.48), and (3.5). We do not actually have to solve Eqs. (3.23)-(3.26) directly. The equations for $X^{\alpha}(\sigma)$ enter as identities and the need to solve them for $P_{\alpha}$ is obviated once we have imposed the constraints (3.5), which fix the embedding momenta as functionals of the string variables and the embeddings. This can be done in terms of the initial data and we are guaranteed that the constraints will continue to hold throughout the evolution. Thus the initial data set can be viewed as a specification of data for the string variables on some initial Cauchy surface specified by $\bar{X}^{\alpha}$.

Although the remaining equations (3.27)-(3.30) may look rather formidable, their general solution can be found quite easily from the following considerations. A solution to (3.27)-(3.30) amounts to a specification of the values of the string variables on a Cauchy surface chosen by the embedding variables. We obtain a solution for $x^{\mu}$ by restricting the known solution in conformal coordinates to this embedding. The solutions for the remaining variables, $p_{\mu}$ in particular, can then be extracted from the functional Hamiltonian equations.

The general space-time solution for $x^{\mu}$ is given by 


$$
x^{\mu}\left(\mathrm{X}^{+}, \mathrm{X}^{-}\right)=f^{\mu}\left(\mathrm{X}^{+}\right)+g^{\mu}\left(\mathrm{X}^{-}\right)
$$

where

$$
f^{\mu}\left(\mathrm{X}^{+}+2 \pi\right)+g^{\mu}\left(\mathrm{X}^{-}-2 \pi\right)=f^{\mu}\left(\mathrm{X}^{+}\right)+g^{\mu}\left(\mathrm{X}^{-}\right) \text {. }
$$

Upon restriction of this solution to an embedding, we obtain the canonical solution as a function of $\sigma$ and a functional of $X$ :

$$
x^{\mu}[X](\sigma)=f^{\mu}\left(X^{+}(\sigma)\right)+g^{\mu}\left(X^{-}(\sigma)\right) .
$$

We substitute (3.31) into (3.27) or (3.28) and extract the solution for the string momenta

$$
\begin{aligned}
p_{\mu}[X](\sigma)= & \eta_{\mu \nu}\left[X^{+}{ }_{, 1} f^{\nu}{ }_{+}\left(X^{+}(\sigma)\right)\right. \\
& \left.-X^{-}{ }_{, 1} g_{,-}^{\nu}\left(X^{-}(\sigma)\right)\right] .
\end{aligned}
$$

The solutions for $x^{\mu}$ and $p_{\mu}$ are to be matched to the initial data $\left(\bar{x}^{\mu}, \bar{p}_{\mu}\right)$ on the initial embedding $\bar{X}^{\alpha}$. If desired, the resulting solutions can be expressed in terms of an arbitrary system of coordinates $\mathrm{X}^{\alpha^{\prime}}=\mathrm{X}^{\alpha^{\prime}}(\mathrm{X})$ by performing the point canonical transformation:

$$
\begin{aligned}
& X^{\alpha}(\sigma) \rightarrow X^{\alpha^{\prime}}(\sigma):=X^{\alpha^{\prime}}(X(\sigma)), \\
& P_{\alpha}(\sigma) \rightarrow P_{\alpha^{\prime}}(\sigma)=\left.\frac{\partial \mathrm{X}^{\beta}}{\partial \mathrm{X}^{\alpha^{\prime}}}\right|_{\mathrm{X}=X(\sigma)} P_{\beta}(\sigma) .
\end{aligned}
$$

The string solutions, constructed in the manner outlined above, reflect the underlying diffeomorphism invariance of the theory through the appearance of two arbitrary functions, namely, the embeddings $X^{\alpha}(\sigma)$. From the perspective of Hamiltonian dynamics on $\Gamma^{\prime}$, these functions simply serve to select the arbitrary embedding on which we measure the fields. If we $f i x$ a one-parameter family of embeddings, i.e., a foliation, with which to describe the dynamics, the solutions to the equations of motion become unique. If we wish to fix only the embedding velocities, then there may still exist residual arbitrariness. For example, in the "conformal gauge"

$$
N=1, \quad N^{1}=0,
$$

we still have the freedom to redefine the foliation by the induced action of a conformal isometry.

\section{B. Harmonic gauge}

Our interest in using the harmonic gauge to represent $\operatorname{diff}(M)$ stems from a question posed in Ref. 2 concerning the feasibility of the corresponding approach in canonical gravity. Recently, this gauge has also attracted some interest in studies of string quantum mechanics and string field theory. ${ }^{7}$ From the string perspective, the utility of the harmonic gauge lies in the fact that, like the conformal gauge, it respects both target space Poincaré invariance and the ever important Weyl invariance.

The harmonic gauge can be specified in terms of the auxiliary structure as

$$
g^{a b} \nabla_{a} \nabla_{b} Y^{(\alpha)}=0 .
$$

For a fixed choice of $Y$, Eq. (3.33) is again to be interpreted as a pair of restrictions on allowable metrics. This way of gauge fixing differs from the conformal gauge in that the metric is related to the auxiliary structure through an expression involving (in particular time) derivatives of the metric. Hence one can say that metric fixing occurs dynami- cally. As always, we can put (3.33) into a more conventional form by choosing coordinates on $M$ adapted to the auxiliary foliation: This yields

$$
(1 / \sqrt{-g}) \partial_{(\beta)}\left(\sqrt{-g} g^{(\alpha)(\beta)}\right)=0
$$

From this expression of the gauge-fixing condition it is clear that the conformal gauge is a special case of the harmonic gauge.

It would be rather awkward to try and extract the functional dependence of $g_{a b}$ on $Y$ directly from (3.33). Because (3.33) can be interpreted as a dynamical equation, it is most expeditious to incorporate the gauge-fixing condition at the level of the action principle by adding a gauge-fixing term to the action. (This, of course, could also have been done in the case of the conformal gauge, but such a scheme is excessively elaborate.) We thus begin with the following gauge-fixed action:

$$
\begin{aligned}
S[x, g, \lambda]= & -\int_{M} \sqrt{-g} g^{a b}\left(\frac{1}{2} \eta_{\mu \nu} \nabla_{a} x^{\mu} \nabla_{b} x^{\nu}\right. \\
& \left.+\nabla_{a} \lambda_{(\alpha)} \nabla_{b} Y^{(\alpha)}\right)
\end{aligned}
$$

where $\lambda_{(\alpha)}$ are Lagrange multipliers to be viewed as a pair of scalar functions on $M$. This action, while defined in a geometric (i.e., coordinate independent) manner, is not invariant with respect to diffeomorphisms. This is because we are not free to redefine the fixed maps $Y^{(\alpha)}$ by using the pullback action of the diffeomorphisms. This is completely analogous to the lack of diffeomorphism invariance of the action (2.1), where it is the metric which is to be immutable.

The equations of motion associated with (3.34) are

$$
\begin{gathered}
\frac{\delta S}{\delta x^{\mu}}=\eta_{\mu \nu} \sqrt{-g} g^{a b} \nabla_{a} \nabla_{b} x^{\nu}=0, \\
\frac{2}{\sqrt{-g}} \frac{\delta S}{\delta g_{a b}}=\left(\delta_{(c}^{a} \delta_{d)}^{b}-\frac{1}{2} g^{a b} g_{c d}\right)\left(\eta_{\mu \nu} \nabla^{c} x^{\mu} \nabla^{d} x^{\nu}\right. \\
\left.+2 \nabla^{c} \lambda_{(\alpha)} \nabla^{d} Y^{(\alpha)}\right)=0,
\end{gathered}
$$

and

$$
\frac{\delta S}{\delta \lambda_{(\alpha)}}=\sqrt{-g} g^{a b} \nabla_{a} \nabla_{b} Y^{(\alpha)}=0
$$

We cannot obtain a dynamical equation for $\lambda_{(\alpha)}$ directly from the action because $Y^{(\alpha)}$ is not to be varied. Nevertheless, an equation of motion for the Lagrange multipliers can be obtained by differentiating (3.36) and using (3.35) and (3.37). We obtain

$$
\left(\nabla^{a} \nabla_{a} \lambda_{(\alpha)}\right) \nabla_{b} Y^{(\alpha)}=0 .
$$

The covectors $\nabla_{b} Y^{(\alpha)}$ constitute a one-form basis for $M$. Hence (3.38) is satisfied if and only if

$$
\nabla^{a} \nabla_{a} \lambda_{(\alpha)}=0
$$

Equations (3.35), (3.37), and (3.39) are the dynamical equations in the harmonic gauge; these equations must be solved subject to the constraints (3.36). If the constraints (3.36) are satisfied on a Cauchy surface, they will continue to be satisfied throughout the dynamical evolution. The physical subset of string solutions is obtained by choosing vanishing Cauchy data for the multipliers. 
As usual, the first step on the road to the parametrized Hamiltonian formalism is to introduce a foliation $X^{\alpha}(\sigma, \tau)$. The action is pulled back to $R \times S^{1}$, yielding

$$
\begin{aligned}
S[x, g, \lambda]= & \int_{R \times S^{1}}\left[\frac{1}{2} N^{-1}\left(\dot{x}^{\mu}-N^{1} x^{\mu}{ }_{, 1}\right)\left(\dot{x}_{\mu}-N^{1} x_{\mu, 1}\right)\right. \\
& +N^{-1} Y^{(\alpha)}{ }_{\beta}\left(\dot{\lambda}_{(\alpha)}-N^{1} \lambda_{(\alpha), 1}\right) \\
& \times\left(\dot{X}^{\beta}-N^{1} X^{\beta}{ }_{, 1}\right)-N\left(\frac{1}{2} x^{\mu}{ }_{, 1} x_{\mu, 1}\right. \\
& \left.\left.+Y^{(\alpha)}{ }_{\beta} \lambda_{(\alpha), 1} X^{\beta}{ }_{, 1}\right)\right] .
\end{aligned}
$$

The definition of the string momenta that follows from (3.40) is the usual one:

$$
p_{\mu}=N^{-1}\left(\dot{x}_{\mu}-N^{1} x_{\mu, 1}\right) \text {. }
$$

The momenta $\mu^{(\alpha)}$ conjugate to the multipliers are defined by

$$
\mu^{(\alpha)}=N^{-1} Y_{, \beta}^{(\alpha)}\left(\dot{X}^{\beta}-N^{1} X^{\beta}{ }_{, 1}\right) .
$$

Prior to parametrization, $X^{\alpha}(\sigma, \tau)$ is a fixed map from $R \times S^{1}$ to $M$. Equation (3.41) therefore represents a constraint. If we also define the momenta conjugate to the lapse and shift, we find that they vanish-another pair of constraints. The combined set of constraints is easily verified to be second class. We need not, however, go through the full Dirac bracket procedure for these constraints: They arise simply because the action (3.40) is already in Hamiltonian form with respect to the multipliers $\lambda_{(\alpha)}$ and their conjugate momenta which, from (3.41), are combinations of the lapse and shift. Hence we need only perform the Legendre transformation in the string sector to obtain the phase space action:

$$
\begin{aligned}
S[x, p, \lambda, \mu]= & \int_{R \times S^{1}}\left[p_{\mu} \dot{x}^{\mu}+\mu^{(\alpha)} \dot{\lambda}_{(\alpha)}\right. \\
& -N\left(\mathscr{H}+Y^{(\alpha)}{ }_{, \beta} \lambda_{(\alpha), 1} X^{\beta}{ }_{, 1}\right) \\
& \left.-N^{1}\left(\mathscr{H}_{1}+\mu^{(\alpha)} \lambda_{(\alpha), 1}\right)\right] .
\end{aligned}
$$

In (3.42) we are to think of the lapse and shift as being fixed functionals of the momenta $\mu^{(\alpha)}$, obtained by inverting (3.41). We will not need the explicit form of these functionals in what follows. Note that the lapse and shift no longer play the role of multipliers enforcing the constraints (2.47) and (2.48): They now enter as dynamically determined degrees of freedom. Of course, this is because we have fixed the gauge. In fact, there are no constraints associated with (3.42); this corresponds to the expected loss of diffeomorphism invariance.

To regain diffeomorphism invariance and to achieve our goal of representing $\operatorname{diff}(M)$, we should parametrize the theory. To do this, we start again with (3.40), but now view $X^{(\alpha)}(\sigma, \tau)$ as a dynamical variable. The definition (3.41) no longer represents a constraint and we have the additional set of momenta conjugate to the embeddings defined by

$$
P_{\alpha}=N^{-1} Y_{, \alpha}^{(\beta)}\left(\dot{\lambda}_{(B)}-N^{1} \lambda_{(B), 1}\right) \text {. }
$$

The phase space of the parametrized string is now doubly extended: We shall denote it by $\Gamma^{\prime \prime}$ :

$$
\begin{aligned}
\Gamma^{\prime \prime}= & T^{*}\left[E m b_{\eta}\left(S^{1}, M^{d}\right)\right. \\
& \left.\times C^{\infty}\left(S^{1}, R^{2}\right) \times E m b_{g}\left(S^{1}, M\right)\right] .
\end{aligned}
$$

The three factors making up $\Gamma^{\prime \prime}$ correspond to the string, multiplier, and embedding subspaces, respectively. The fundamental Poisson brackets are as before for the string and embedding variables. For the multipliers we have

$$
\left[\lambda_{(\alpha)}(\sigma), \mu^{(\beta)}\left(\sigma^{\prime}\right)\right]=\delta_{(\alpha)}^{(\beta)} \delta\left(\sigma, \sigma^{\prime}\right)
$$

The action functional for the doubly extended phase space takes the form

$$
\begin{aligned}
& S[x, p, \lambda, \mu, X, P, \vec{N}] \\
& =\int_{R \times S^{\prime}}\left(p_{\mu} \dot{x}^{\mu}+\mu^{(\alpha)} \dot{\lambda}_{(\alpha)}\right. \\
& \left.\quad+P_{(\alpha)} \dot{X}^{\alpha}-N \mathbf{H}^{\prime \prime}-N^{\prime} \mathbf{H}_{1}^{\prime \prime}\right)
\end{aligned}
$$

where

$$
\begin{aligned}
& \mathbf{H}^{\prime \prime}=\mathscr{H}+\left(Y^{-1}\right)_{(\beta)}^{\alpha} P_{\alpha} \mu^{(\beta)}+Y_{, \beta}^{(\alpha)} \lambda_{(\alpha), 1} X_{, 1}^{\beta} \approx 0, \\
& \mathbf{H}_{1}^{\prime \prime}=\mathscr{H}_{1}+\mu^{(\alpha)} \lambda_{(\alpha), 1}+P_{\alpha} X^{\alpha}{ }_{, 1} \approx 0 .
\end{aligned}
$$

In $(3.43 \mathrm{a})$ we have defined $\left(Y^{-1}\right)_{(\beta)}^{\alpha}$ to be the inverse of $Y_{, \beta}^{(\alpha)}$;

$$
\left(Y^{-1}\right)_{(\beta)}^{\alpha} Y_{, \gamma}^{(\beta)}=\delta_{\gamma}^{\alpha}
$$

Both $Y^{(\alpha)}{ }_{\beta}$ and $\left(Y^{-1}\right)_{(\beta)}^{\alpha}$ are fixed functionals of the embeddings.

As a result of the parametrization process, the role of the lapse and shift has undergone something of a metamorphosis. This is most easily understood when we observe that through (2.9)-(2.11), the lapse and shift are functionals of both the space-time metric and the foliation $X$. Prior to the parametrization, the foliation is fixed and hence in (3.42) the lapse and shift are the (dynamically determined) descriptors of the harmonic gauge foliation. By including the foliation (or rather the embeddings) into the phase space, the lapse and shift are no longer locked into the space-time metric, but rather become as freely variable as the foliation. The lapse and shift then resume their role as nondynamical Lagrange multipliers enforcing the constraints (3.43). These constraints (3.43) represent the reintroduction of diffeomorphism invariance- the desired result of the parametrization process. As they should be, the constraints are first class: They in fact obey a Dirac algebra identical to (2.54)(2.56).

From our work above, we know that in order to represent diff $(M)$ we must Abelianize the constraint algebra by effectively unprojecting the constraint functions in (3.43). This amounts to finding (nondegenerate) combinations of $\mathbf{H}^{\prime \prime}$ and $\mathbf{H}_{1}^{\prime \prime}$ which isolate the embedding momenta as, for example, in (3.5). While we could solve the constraints for the embedding momenta by "brute force," it is more instructive to do this by extracting from $\Gamma$ " the "hypersurface basis" and its dual. The hypersurface basis is an orthonormal frame at each point of a given embedding. One leg of the dyad is the unit normal to the embedded circle and the other leg is the unit tangent. The quickest way to find this basis is to use the dynamical equation for $X^{\alpha}$ :

$$
\dot{X}^{\alpha}=N\left(Y^{-1}\right)_{(\beta)}^{\alpha} \mu^{(\beta)}+N^{1} X^{\alpha}, 1 .
$$

By comparing (3.44) with (2.9) we can read off the unit normal, 


$$
n^{\alpha}=\gamma^{-1 / 2}\left(Y^{-1}\right)_{(\beta)}^{\alpha} \mu^{(\beta)}
$$

and the unit tangent,

$$
t^{\alpha}=\gamma^{-1 / 2} X^{\alpha}{ }_{11} .
$$

In order to obtain the Abelian constraints we will need to construct the corresponding dual basis. This can be done by choosing an arbitrary volume form $\epsilon_{a b}$ on $\boldsymbol{M}$. The normal covector is defined as

$$
m_{\alpha}:=J^{-1} \gamma^{1 / 2} \epsilon_{\alpha \beta} X^{\beta}{ }_{, 1}=-n_{\alpha},
$$

where

$$
J=\epsilon_{\alpha \beta}\left(Y^{-1}\right)_{(\gamma)}^{\alpha} X^{\beta}{ }_{, 1} \mu^{(\gamma)} .
$$

The spatial leg of the covector basis is then

$$
t_{\alpha}:=J^{-1} \gamma^{1 / 2} \epsilon_{\beta \alpha}\left(Y^{-1}\right)_{(\gamma)}^{\beta} \mu^{(\gamma)}=\gamma^{1 / 2} X_{\alpha}^{1} .
$$

It is straightforward to check that the vector and covector bases are dual.

Note that in the definition of the covector basis it was necessary that $J$ be nonvanishing. Inspection of (3.41) reveals that this simply means that the deformation vector is never tangent to a given embedding. Alternatively, the map $X^{\alpha}(\sigma, \tau)$, defined by the dynamical data (in a given coordinate patch) through (3.44), must be a diffeomorphism. The nonvanishing of $J$ is thus guaranteed by our various hypotheses, but it is important to realize that this requirement does place restrictions on admissible initial data. With this proviso understood, we can continue.

Crucial for the work to follow is the fact that the covector basis is defined independent of the choice of volume form on $M$. This is because the space of two-forms (at a point) is one-dimensional. The dependence of the definitions (3.47)(3.49) on the choice of volume form drops out because only ratios of terms linear in $\epsilon_{a b}$ are used. The vector basis and its dual are thus fixed functionals on the phase space $\Gamma^{\prime \prime}$. (Strictly speaking, the normalized basis depends also on the induced metric $\gamma$. As usual, we need not consider this function as an element of the phase space. Weyl invariance will guarantee its absence in any of our final expressions.) This result, ultimately following from (3.44), relies crucially on the gauge-fixing procedure. Without it, we would have no way of extracting the hypersurface basis.

Armed with the hypersurface covector basis, we construct the unprojected constraints:

$$
\mathbf{H}_{\alpha}^{\prime \prime}=-\gamma^{-1 / 2} n_{\alpha} \mathbf{H}^{\prime \prime}+X_{\alpha}^{1} \mathbf{H}_{1}^{\prime \prime}=P_{\alpha}+\mathscr{H}_{\alpha}^{\prime \prime} \approx 0,
$$

where

$$
\begin{aligned}
\mathscr{H}_{\alpha}^{\prime \prime}= & \mathscr{H}_{\alpha}-\gamma^{-1 / 2} n_{\alpha} Y^{(\beta)}{ }_{, \gamma} \lambda_{(\beta), 1} \\
& \times X^{\gamma}{ }_{1}+X_{\alpha}^{1} \mu^{(\beta)} \lambda_{(\beta), 1} .
\end{aligned}
$$

Using (3.47)-(3.49) it can be verified that the constraints (3.50) are independent of $\gamma$. That the constraints obey an Abelian Poisson algebra

$$
\left[\mathbf{H}_{\alpha}^{\prime \prime}(\sigma), \mathbf{H}_{\beta}^{\prime \prime}\left(\sigma^{\prime}\right)\right]=0
$$

is seen by using an argument which apparently goes back to Dirac. $^{8}$ Since the constraints in (3.50) are combinations of the original first class constraints, they will also be first class. When we compute the Poisson bracket between two of the constraint functions ( 3.50 ), the result will be independent of the embedding momenta $P_{\alpha}$ because the embedding momenta only appear in the single term shown in (3.50). This is consistent with the first class nature of $\mathbf{H}_{\alpha}^{\prime \prime}$ if and only if (3.51) holds.

For completeness, let us point out that there is an alternate route to the unprojected constraints. As we did for the parametrized scalar fields, we could rewrite (3.42) in a form that is linear in the embedding velocites $\dot{X}^{\alpha}$. To do this we would need to "unproject" the lapse and shift by extracting from (3.41) [which is equivalent to (3.44)] the hypersurface basis. Upon adjoining the embeddings to the phase space, the definitions of the conjugate momenta $P_{\alpha}$ would appear as the constraints $\mathbf{H}_{\alpha}^{\prime \prime} \approx 0$.

By using either of these two equivalent methods, our success in extracting the unprojected constraints means success in representing $\operatorname{diff}(M)$. The homomorphic mapping from $\operatorname{diff}(M)$ into the Poisson algebra of functions on $\Gamma^{\prime \prime}$ is given by

$$
V \rightarrow \vec{V} \rightarrow \mathbf{H}^{\prime \prime}(\vec{V}),
$$

where

$$
\mathbf{H}^{\prime \prime}(\vec{V})=\int_{S^{\prime}} V^{\alpha}(X(\sigma)) \mathbf{H}_{\alpha}^{\prime \prime} .
$$

Given two elements of $\operatorname{diff}(M)$, i.e., given two complete vector fields $\vec{U}$ and $\vec{V}$ on $M$, we have

$$
\left[\mathbf{H}^{\prime \prime}(\vec{U}), \mathbf{H}^{\prime \prime}(\vec{V})\right]=\mathbf{H}^{\prime \prime}(-[\vec{U}, \vec{V}]) .
$$

Using the fact that conformal gauge metrics are also harmonic gauge metrics, the subalgebra conf $(M, g)$ can be represented as in Sec. III A.

As in the case of the conformal gauge, the dynamical evolution generated by $\mathbf{H}^{\prime \prime}(\vec{V})$ builds a physically acceptable solution to (3.35) $-(3.37)$ provided that we restrict the allowable initial data. In the present situation this is most easily done by imposing, in addition to (3.50), the following constraint:

$$
\lambda_{(\alpha)} \approx 0 .
$$

The demand that (3.53) be preserved by the dynamical evolution implies that

$$
P_{\alpha} \approx 0 \text {, }
$$

as can be seen, e.g., by taking the Poisson bracket of (3.53) with the Hamiltonian

$$
\mathbf{H}^{\prime \prime}(\vec{N}):=\int_{S_{1}}\left(N \mathbf{H}^{\prime \prime}+N^{1} \mathbf{H}_{1}^{\prime \prime}\right) .
$$

It is easily checked in a similar fashion that ( 3.54 ) is automatically preserved in time (modulo the constraints). The complete set of constraints (3.50), (3.53), and (3.54) are thus first class, hence they are satisfied for all time if satisfied initially. Moreover, these constraints imply that the string initial data satisfy the original constraints $(2.47)$ and (2.48). The representatives $\mathbf{H}^{\prime \prime}(\vec{V})$ thus evolve the physical string initial data into physical string solutions. 


\section{REPRESENTATION OF diff ( $M$ ) ON THE ORIGINAL PHASE SPACE}

As the Hamiltonian structure of general relativity began to be unraveled in the late 1950's and early 1960's, ${ }^{9}$ it became clear that the contraints that arise in generally covariant theories are due to the fact that somehow time and space coordinates are camouflaged within the original dynamical variables. More precisely, the location of a spacelike hypersurface (time) and coordinates on it (space) are implicitly contained in the phase space variables. The momenta conjugate to these variables must then be identified with energy and momentum densities; these identifications are the Hamiltonian and momentum constraints. A clean example of this structure is provided by the parametized harmonic maps considered previously. There, the time and space coordinates, i.e., the embeddings, were easy to identify since they were essentially put in by hand.

The outstanding challenge in systems that are "already parametrized," such as general relativity and the relativistic string, is to extract the embeddings directly from the original phase space. In general relativity this challenge has never been fully met. It may be that for this theory (and perhaps more generally) there is no single identification of embedding variables suitable for all situations. The relative simplicity of the string permits one to go much further, there is a rather natural choice of embeddings. Perhaps not unexpectedly, the rewriting of string theory as a parametrized theory is closely tied to - and rests on the validity of - the light-cone gauge. Having explicitly exposed the parametrized aspects of the string, we once again will have succeeded in representing $\operatorname{diff}(M)$.

Our method of expressing the string as a parametrized system is based on the observation made by Kucharr ${ }^{10}$ that there is a canonical transformation which takes the (projected) constraints of a parametrized scalar field on a two-dimensional Minkowski space-time into a form identical to those of the string. Here we will essentially run that argument backward. Begin by defining two covariantly constant, linearly independent null vectors $k^{\mu}$ and $l^{\mu}$ in the target space $M^{a}$ :

$$
l_{\mu} l^{\mu}=0=k^{\mu} k_{\mu}
$$

they are to be normalized relative to each other such that

$$
\eta_{\mu v} k^{\mu} l^{v}=-1 \text {. }
$$

Light-cone components for any objects with target space (Lorentz) indices are defined using $k^{\mu}, l^{\mu}$; in particular,

$$
x^{+}:=-\eta_{\mu \nu} x^{\mu} l^{\nu}, x^{-}:=-\eta_{\mu \nu} x^{\mu} k^{\nu},
$$

and

$$
p_{+}:=k^{\mu} p_{\mu}, p_{-}:=l^{\mu} p_{\mu} .
$$

In terms of light-cone components the string constraints take the form

$$
\begin{aligned}
& \mathscr{H}=-p_{+} p_{-}-x^{+}{ }_{, 1} x^{-}{ }_{, 1}+\frac{1}{2}\left(p_{i} p^{i}+x^{i}{ }_{1,} x_{i, 1}\right) \approx 0, \\
& \mathscr{H}_{1}=p_{+} x^{+}{ }_{, 1}+p_{-} x^{-}{ }_{, 1}+p_{i} x_{, 1}^{i} \approx 0 .
\end{aligned}
$$

Latin indices from the middle of the alphabet are taken to label spacelike directions in $M^{d}$ which are orthogonal to the null vectors, i.e., $i=1,2, \ldots, d-2$.
Now, consider the transformation defined by

$$
\begin{aligned}
& T_{, 1}=-\left(p_{-}\right)_{0}^{-1} x^{+}{ }_{, 1}, \\
& S_{, 1}=\left(p_{-}\right)_{0}^{-1} p_{-}, \\
& P_{T}=-\left(p_{-}\right)_{0} p_{+}, \\
& P_{S}=\left(p_{-}\right)_{0} x^{-},
\end{aligned}
$$

where

$$
\left(p_{\mu}\right)_{0}:=\frac{1}{2 \pi} \int_{S_{1}} p_{\mu} .
$$

Our reuse of the symbols $T$ and $S$ will be justifed shortly. In terms of the relabeled variables, (4.1) and (4.2) become

$$
\begin{aligned}
& \mathscr{H}=P_{T} S_{, 1}+P_{S} T_{, 1}+\frac{1}{2}\left(p_{i} p^{i}+x_{, 1}^{i} x_{i, 1}\right) \approx 0, \\
& \mathscr{H}_{1}=P_{T} T_{, 1}+P_{S} S_{, 1}+p_{i} x_{, 1}^{i} \approx 0 .
\end{aligned}
$$

Comparing with (2.36) and (2.37), we see that Eqs. (4.7) and (4.8) are precisely the form of the constraints that arise in the parametrized formalism for a set of $d-2$ scalar fields $x^{i}$ in two dimensions. This interpretation of (4.7) and (4.8) has the variables $T(\sigma)$ and $S(\sigma)$ playing the role of spacelike embeddings with respect to conformal coordinates on $M$. In contrast to our previous work with the conformal gauge (III), no gauge fixing is needed here to provide a privileged set of coordinates. The string itself determines a conformal coordinate system (up to a choice of origin; see the discussion below).

At this point, our identification of the string as a set of $d-2$ parametrized scalar fields has been purely formal. A closer examination of the transformation (4.3)-(4.6) is warranted. To begin, the transformation, strictly speaking, is not canonical on $\Gamma$. On the circle the operator $\partial_{\sigma}$ has a nonempty kernel given by the constant functions. In particular, the mapping $\left(x^{-}, p_{-}\right) \leftrightarrow\left(S, P_{S}\right)$ is not bijective. (This issue did not arise in Ref. 10 because there $M$ was taken to be an infinite two-dimensional Minkowski space.) Thus for example, $P_{S}$ as defined in (4.6) has a vanishing constant mode:

$$
\int_{S^{\prime}} P_{S}=\left(p_{-}\right)_{0} \int_{S^{\prime}} x^{-}, 1=0 .
$$

As a result, the variables $S(\sigma)$ and $P_{S}(\sigma)$ defined through (4.4) and (4.6) cannot be canonically conjugate; if they were, we would obtain a contradiction:

$$
1=\int_{0}^{2 \pi} d \sigma^{\prime} \delta\left(\sigma, \sigma^{\prime}\right)=\left[S(\sigma), \int_{S^{\prime}} P_{S}\right]=0 .
$$

There is, however, a natural subspace of $\Gamma$ for which the transformation (4.3)-(4.6) is canonical. This subspace is obtained by effectively factoring out the "center of mass" portion of $\Gamma$. Denote by $\widetilde{\Gamma}$ the cotangent bundle over the space $\widetilde{E} m b_{\eta}\left(S^{1}, M^{d}\right)$ of based loops in $M^{d}$, i.e., embeddings of $S^{1}$ into $M^{d}$ which begin and end at a fixed point. As a differentiable manifold, $\widetilde{E} m b_{\eta}\left(S^{1}, M^{d}\right)$ has many nice properties, e.g., it is a Kähler manifold. "We can identify $\widetilde{E} m b_{\eta}\left(S^{1}, M^{d}\right)$ with the space of exact one-forms on $S^{1}$ taking their values in Minkowski vector space. The string phase space can then be decomposed as 


$$
\begin{aligned}
\Gamma & \simeq T^{*} M^{d} \times \widetilde{\Gamma} \\
& \simeq M^{d} \times M^{d} \times T^{*} \widetilde{E} m b_{\eta}\left(S^{1}, M^{d}\right) .
\end{aligned}
$$

For a given value of $\left(p_{\mu}\right)_{0}$ local (noncanonical) coordinates for $\widetilde{\Gamma}$ are provided by $\left(x^{\mu}, 1, p_{v}\right)$ or, alternatively, $\left(\alpha^{\mu}, \widetilde{\alpha}^{\nu}\right)$ [defined in (3.21) and (3.22)]. It is a key feature of the string constraints [e.g., (4.1) and (4.2) or (4.7) and (4.8)] that they are essentially only functions on $\widetilde{\Gamma}$. In any computations we will perform which involve only the constraints, the center of mass momenta $\left(p_{\mu}\right)_{0}$ will simply "go along for the ride."

The transformation (4.3)-(4.6) can be interpreted as a one-parameter family of canonical transformation on $\widetilde{\Gamma}$, where the parameter is $\left(p_{-}\right)_{0}$. This parameter represents the discontinuity which $S(\sigma)$ must possess if it is to be the spatial coordinate location of an embedded circle. For this reason $-\left(p_{-}\right)_{0}$ is often called the "string length" in the light-cone gauge; see the discussion below. We have rescaled the would-be embedding variables so that this discontinuity has the value $2 \pi$. Thus if $\sigma \in[0,2 \pi]$, with $\sigma=0$ and $\sigma=2 \pi$ identified, we have

$$
S(2 \pi)-S(0)=\int_{0}^{2 \pi} d \sigma S_{, 1}=2 \pi .
$$

We must then make the identification

$$
S \sim S+2 \pi
$$

to produce embeddings with the topology of a circle.

While we have uncovered the natural space on which the transformation is bijective, there is still the problem that the embeddings and their conjugate momenta as identified in (4.3)-(4.6) do not constitute a canonical chart on $T^{*} E m b\left(S^{1}, M\right)$. At the root of this difficulty is the degeneracy of the transformations (4.4) and (4.6), which reflects the fact that the light-cone string variables do not contain all of the "pure gauge" degrees of freedom. To see this, notice that (4.4) leaves a mode of $S(\sigma)$ unspecified. This mode can be taken to represent a choice of origin for the $S$ coordinate relative to the $\sigma$ origin. It is not fixed by the light-cone variables, nor is it fixed by the constraints (4.7) and (4.8). The freedom to choose the origin for $S$ is a miniature gauge freedom introduced by the degenerate nature (on $\Gamma$ ) of the transformations. Indeed, the corresponding first class constraint on $T^{*} E m b\left(S^{1}, M\right)$ is simply

$$
\int_{S^{\prime}} P_{S} \approx 0
$$

Alternatively, by virtue of the constrainsts (4.7) and (4.8), (4.10) can be viewed as a restriction on allowable initial data for the transverse string variables:

$$
\begin{gathered}
\int_{S^{\prime}}\left[\left(S_{, 1}\right)^{2}-\left(T_{, 1}\right)^{2}\right]^{-1}\left[\frac { 1 } { 2 } T _ { , 1 } \left(p_{i} p^{i}\right.\right. \\
\left.\left.+x_{, 1}^{i} x_{i, 1}\right)-S_{, 1}\left(p_{i} x_{, 1}^{i}\right)\right] \approx 0 .
\end{gathered}
$$

As we shall see shortly, this constraint is the precursor of the familiar shift of origin constraint which remains after going to the light-cone gauge. ${ }^{4}$

The single degree of freedom that is fixed by the constraint (4.11) can be thought of as the missing constant mode of the embedding momentum $\boldsymbol{P}_{S}$. Its conjugate coordinate is to be a mode of $S(\sigma)$ such that the embeddings and their momenta provide a true canonical chart. Evidently, extracting this single remaining degree of freedom from the transverse subspace of the phase space is an unpleasant task and we have not succeeded in doing this in any nice way. We can sidestep this difficulty by using the same strategy that served us so well in Sec. III: If there is difficulty in isolating the embeddings from the original phase space, simply introduce them explicity. We do this as follows. Fix the origin of $S$ relative to $\sigma$ by integrating (4.4) as

$$
S(\sigma)=\left(p_{-}\right)_{0}^{-1}\left(-q+\int_{0}^{\sigma} d x p_{-}(x)\right) .
$$

We now treat $q$ as a dynamical variable. Its conjugate momentum, denoted $p$, must be constrained to vanish:

$$
p \approx 0,
$$

so that $q$ is pure gauge, i.e., not dynamically determined; hence the enlarged phase space has the same physical content as before. The momentum is incorporated into the definition of $P_{S}$ via

$$
P_{S}=\left(p_{-}\right)_{0}\left(x^{-}, 1-p \delta(\sigma)\right)
$$

$P_{S}$ is thus weakly equal to its original definition. (Note that $P_{S}$ is now a distribution. This feature of the formalism can be avoided by introducing an auxiliary prescribed measure on $S^{1}$. For simplicity we will retain the delta function "measure" in what follows.) Using the Poisson bracket

$$
[q, p]=1 \text {, }
$$

it is easily verified that $S(\sigma)$ and $P_{S}(\sigma)$ are canonically conjugate variables:

$$
\left[S(\sigma), P_{S}\left(\sigma^{\prime}\right)\right]=\delta\left(\sigma, \sigma^{\prime}\right) .
$$

The remaining degree of freedom in the light-cone subspace of $\Gamma$ corresponds to the coordinate conjugate to the string length $\left(p_{-}\right)_{0}$. Intuitively, this single mode should represent the constant map from $S^{1}$ into the " - " direction of $M^{d}$. To extract this mode from $x^{-}(\sigma)$ we need to choose a measure on the circle. A natural choice is given by $S_{, 1}$, as defined in (4.4). This choice of measure "weighs" functions using the metric induced on the circles $T=$ const by a flat metric on $M$. Using this measure to define the homogeneous mode of $x^{-}$and then extending the definition to provide a canonical coordinate on the extension of $\Gamma$, we obtain

$$
\left(x^{-}\right)_{0}:=\left(p_{-}\right)_{0}^{-1} \int_{S_{1}}\left(p_{-} x^{-}-p_{+} x^{+}-\frac{q p}{2 \pi}\right) .
$$

Straightforward computation confirms that $\left(x^{-}\right)_{0}$ has vanishing Poisson brackets with all variables except $\left(p_{-}\right)_{0}$ : This bracket is

$$
\left[\left(x^{-}\right)_{0},\left(p_{-}\right)_{0}\right]=1 \text {. }
$$

To summarize, if we work on a slightly extended phase space $\Gamma^{*}$ obtained by including the canonical pair $q, p$, then from the light-cone string variables we can isolate a natural set of embedding variables (relative to a conformal coordinate chart on $M$ ) by the canonical transformation on $\Gamma^{*}$ :

$$
T=-\left(p_{-}\right)_{0}^{-1} x^{+}
$$




$$
\begin{aligned}
& S=-\left(p_{-}\right)_{0}^{-1}\left(q-\int_{0}^{\sigma} d x p_{-}(x)\right) \\
& P_{T}=-\left(p_{-}\right)_{\alpha_{+}} p_{+} \\
& P_{S}=\left(p_{-}\right)_{0}\left(x^{-}, 1-p \delta(\sigma)\right) \\
& \left(x^{-}\right)_{0}=\left(p_{-}\right)_{0}^{-1} \int_{S^{\prime}}\left(p_{--} x^{-}-p_{+} x^{+}-\frac{q p}{2 \pi}\right) \\
& \left(p_{-}\right)_{0}=\frac{1}{2 \pi} \int_{S^{\prime}} p_{-} .
\end{aligned}
$$

In the new canonical variables, the constraints take the form (4.7) and (4.8), along with

$$
\int_{S^{\prime}} P_{S} \approx 0
$$

In (4.7) and (4.8) $P_{S}$ is to be thought of as having all of its modes intact. The transformation (4.13)-(4.18), when complemented with the identity transformation on the transverse phase space, is bijective.

As always, the easiest way to exhibit the desired homomorphisms is through the unprojected (Abelian) constraints. These have already been written (for $d$ scalar fields) in terms of the null combinations of $T$ and $S$ in Sec. III A. Translating (3.20) into the original string variables, we obtain

$$
\begin{aligned}
\mathbf{H}_{+}: & =\frac{1}{2}\left(P_{T}+P_{S}\right)+\left(T_{, 1}+S_{, 1}\right)^{-1} \widetilde{\alpha}_{i} \widetilde{\alpha}^{i} \\
& =\left(p_{-}\right)_{0}\left(\widetilde{\alpha}^{-}-p \delta(\sigma)-\left(2 \widetilde{\alpha}^{+}\right)^{-1} \widetilde{\alpha}_{i} \widetilde{\alpha}^{i}\right) \approx 0
\end{aligned}
$$

and

$$
\begin{aligned}
\mathbf{H}_{-}: & =\frac{1}{2}\left(P_{T}-P_{S}\right)-\left(T_{.1}-S_{, 1}\right)^{-1} \alpha_{i} \alpha^{i} \\
& \left.=(p)_{-}\right)_{0}\left(\alpha^{-}+p \delta(\sigma)-\left(2 \alpha^{+}\right)^{-1} \alpha_{i} \alpha^{i}\right) \approx 0 ;
\end{aligned}
$$

these functions obey an Abelian Poisson algebra.

To construct the representatives of $\operatorname{diff}(M)$ we proceed as follows. Fix a set of coordinates $\mathrm{X}^{ \pm}$on $M$ by letting $\mathrm{X}^{ \pm} \in(-\infty, \infty)$ and making the identification

$$
\mathrm{X}^{+}-\mathrm{X}^{-} \sim \mathrm{X}^{+}-\mathrm{X}^{-}+4 \pi \text {. }
$$

If we define

$$
\begin{aligned}
& X^{ \pm}(\sigma)=(T(\sigma) \pm S(\sigma)), \\
& P_{ \pm}=\frac{1}{2}\left(P_{T} \pm P_{S}\right),
\end{aligned}
$$

then we can identify $X^{ \pm}(\sigma)$ as an embedding of $S^{1}$ into $M$ expressed parametrically in the coordinates $\mathrm{X}^{ \pm}$. The corresponding embedding deformation generators are given in (4.20) and (4.21). Given a vector field $\vec{V}$ on $M$ representing $V \in \operatorname{diff}(M)$, we take its components in the coordinate basis provided by $\mathrm{X}^{ \pm}$and pull the resulting functions $V \pm$ back to $S^{1}$ along the embedding (4.22). We use these two functions on $S^{1}$ to smear the generators $\mathbf{H}_{ \pm}$:

$$
\mathbf{H}(\vec{V})=\int_{S^{\prime}}\left(V^{+} \mathbf{H}_{+}+V^{-} \mathbf{H}_{-}\right),
$$

where the functions $V^{ \pm}(\sigma)$ are to be viewed as fixed functionals of the canonical variables $\left(x^{+}, p_{-}, q\right)$ through the combinations (4.22), (4.13), and (4.14). Either from direct computation or as a consequence of our previous work (III), the map

$$
V \rightarrow \vec{V} \rightarrow \mathbf{H}(\vec{V})
$$

is once again a homomorphism:

$$
[\mathbf{H}(\vec{V}), \mathbf{H}(\vec{W})]=\mathbf{H}(-[\vec{V}, \vec{W}]) .
$$

Notice that the enlargement of $\Gamma$ to include $q$ and $p$ is crucial for this result.

To obtain a representation of $\operatorname{conf}(M, g)$, we need to know how to define conformal Killing vectors. This is slightly subtle since the metric on $M$ is only determined (up to conformal rescalings) by the dynamical evolution of the string initial data. However, having evolved the data, the functions $X^{ \pm}(\sigma)$ have the interpretation of embeddings relative to a null coordinate system on $M$. Hence the restriction of the components of a conformal Killing vector $\vec{v}$ to such an embedding takes the form

$$
v^{ \pm}(\sigma)=v^{ \pm}\left(X^{ \pm}(\sigma)\right) .
$$

The homomorphism from $\operatorname{conf}(M, g)$ into $C^{\infty}\left(\Gamma^{\star}, R\right)$ is given by

$$
v \rightarrow \vec{v} \rightarrow \mathbf{H}(\vec{v}),
$$

where

$$
\mathbf{H}(\vec{v})=\int_{S^{\prime}}\left(v^{+} \mathbf{H}_{+}+v^{-} \mathbf{H}_{-}\right) .
$$

As before, rather than view conf $(M, g)$ as a subgroup of the dynamical group $\operatorname{Diff}(M)$, we can emphasize its role as a symmetry group for the string via the homomorphism

$$
\begin{aligned}
v \rightarrow \vec{v} \rightarrow & \mathbf{P}(\vec{v}), \\
\mathbf{P}(\vec{v})= & \int_{S^{\prime}}\left(p_{-}\right)_{0}\left[v^{+}\left(\tilde{\alpha}^{-}-p \delta(\sigma)\right)\right. \\
& \left.+v^{-}\left(\alpha^{-}+p \delta(\sigma)\right)\right],
\end{aligned}
$$

and the antihomomorphism

$$
\begin{aligned}
v \rightarrow \vec{v} \rightarrow & \mathbf{h}(\vec{v}), \\
\mathbf{h}(\vec{v})= & \int_{S^{\prime}}-\left(p_{-}\right)_{0}\left[v^{-}\left(2 \alpha^{+}\right)^{-1} \alpha_{i} \alpha^{i}\right. \\
& \left.+v^{+}\left(2 \widetilde{\alpha}^{+}\right)^{-1} \widetilde{\alpha}_{i} \widetilde{\alpha}^{i}\right] .
\end{aligned}
$$

Using the fact that $v^{+}(\sigma)$ and $v^{-}(\sigma)$ are fixed functionals of $x^{+}, p_{-}$, and $q$ through the combinations $X^{+}(\sigma)$ and $X^{-}(\sigma)$, respectively, we have

$$
[\mathbf{P}(\vec{v}), \mathbf{P}(\vec{w})]=\mathbf{P}(-[\vec{v}, \vec{w}])
$$

and

$$
[\mathbf{h}(\vec{v}), \mathbf{h}(\vec{w})]=\mathbf{h}([\vec{v}, \vec{w}]) .
$$

As in Sec. III A, the functions $\mathbf{h}(\vec{v})$ are symmetry generators, i.e., constants of motion, while the functions $\mathbf{P}(\vec{v})$ are generators of a conditional symmetry, i.e., they are constants of motion modulo (4.20) and (4.21).

The "diffeomorphism Hamiltonian" $\mathbf{H}(\vec{V})$ generates the dynamical evolution associated with the embedding deformation

$$
X^{\alpha}(\sigma) \rightarrow X^{\alpha}(\sigma)+V^{\alpha}(X(\sigma)),
$$

exactly as in Sec. III A. It is therefore a simple matter to make the identification

$$
X^{ \pm}(\sigma)=-\left(p_{-}\right)_{0}^{-1}\left(x^{+} \pm q \mp \int_{0}^{\sigma} d y p_{-}(y)\right)
$$


and write the solutions to the many-fingered time Hamiltonian equations for the transverse variables. Thus

$x^{i}\left[x^{+}, p_{-}, q\right](\sigma)=f^{i}\left[X^{+}(\sigma)\right]+g^{i}\left[X^{-}(\sigma)\right]$

and

$p_{i}\left[x^{+}{ }_{,} p_{-}, q\right](\sigma)=\delta_{i j}\left(X^{+}{ }_{, 1} f^{j},+-X^{-}{ }_{, 1} g^{j},-\right)$,

where $f^{i}\left(X^{+}\right)$and $g^{i}\left(X^{-}\right)$are any two functions which satisfy

$f^{i}\left(X^{+}+2 \pi\right)+g^{i}\left(X^{-}-2 \pi\right)=f^{i}\left(X^{+}\right)+g^{i}\left(X^{-}\right)$,

are solutions to

$\frac{\delta x^{i}}{\delta X^{ \pm}(\sigma)}=\left[x^{i}, \mathbf{H}_{ \pm}(\sigma)\right], \quad \frac{\delta p_{i}}{\delta X^{ \pm}(\sigma)}=\left[p_{i}, \mathbf{H}_{ \pm}(\sigma)\right]$.

The solutions for the remaining degrees of freedom $\left(x^{-}\right)_{0}$ and $\left(p_{-}\right)_{0}$ are easily obtained by virtue of the fact that they are constants of motion. This can be seen by observing that the coordinates on $\Gamma^{*}$ defined in $(4.13)-(4.18)$ are canonical and in this chart $\mathbf{H}_{ \pm}$are functionals of the embedding and transverse variables only. Thus the functional differential equations for the two remaining phase space coordinates take the simple form

$$
\begin{aligned}
& \frac{\delta\left(x^{-}\right)_{0}}{\delta X^{ \pm}(\sigma)}=\left[\left(x^{-}\right)_{0}, \mathbf{H}_{ \pm}(\sigma)\right]=0, \\
& \frac{\delta\left(p_{-}\right)_{0}}{\delta X^{ \pm}(\sigma)}=\left[\left(p_{-}\right)_{0}, \mathbf{H}_{ \pm}(\sigma)\right]=0 .
\end{aligned}
$$

Solutions to these equations are, of course,

$$
\begin{aligned}
& \left(x^{-}\right)_{0}=a, \\
& \left(p_{-}\right)_{0}=b,
\end{aligned}
$$

where $a$ and $b$ are constants.

To complete the specification of the solutions, we again should match the solutions to an initial data set. This is done as follows. Choose an initial data surface by specifying the initial values $\bar{X}^{\alpha}(\sigma)$ for the embeddings. On that surface specify the initial data $\left[\left(\overline{x^{-}}\right)_{0},\left(\overline{p_{-}}\right)_{0}, \bar{x}^{i}, \bar{p}_{i}\right]$. Initial data for the embedding momenta are fixed by the constraints

$$
\begin{aligned}
& \bar{P}_{+}=-\left(\bar{X}^{+}{ }_{, 1}\right)^{-1} \overline{\widetilde{\alpha}}_{i} \overline{\widetilde{\alpha}}^{i}, \\
& \bar{P}_{-}=\left(\bar{X}^{-}{ }_{, 1}\right)^{-1} \bar{\alpha}_{i} \bar{\alpha}^{i} .
\end{aligned}
$$

Since the constraints are first class, they continue to hold throughout the dynamical evolution; hence (4.33) fixes the evolution of the embedding momenta in terms of the transverse dynamics. The tranverse initial data are also constrained [via (4.10) or (4.11)] [From (4.28), this constraint function represents the action on the transverse string variables of a conformal isometry which is generated by the conformal Killing vector field tangent to the circles $T=$ const $]$ :

$$
\int_{S^{\prime}}\left(\left(\bar{X}^{+}{ }_{, 1}\right)^{-1} \overline{\tilde{\alpha}}_{i} \overline{\tilde{\alpha}}^{i}+\left(\bar{X}^{-}{ }_{, 1}\right)^{-1} \bar{\alpha}_{i} \bar{\alpha}^{i}\right)=0 .
$$

Again, this constraint is first class and need only be satisfied initially. In terms of the original string coordinates, (4.33) and (4.34) are equivalent to

$$
\overline{\widetilde{\alpha}}^{-}=\left(2 \overline{\widetilde{\alpha}}^{+}\right)^{-1} \overline{\widetilde{\alpha}}_{i} \overline{\widetilde{\alpha}}^{i}, \quad \bar{\alpha}^{-}=\left(2 \bar{\alpha}^{+}\right)^{-1} \bar{\alpha}_{i} \bar{\alpha}^{i}, \quad \bar{p}=0 .
$$

With the constraints taken care of, we can now match the solutions to the initial data. For example, let the initial embedding be an element of the preferred foliation associated with the coordinates $X^{\alpha}=(T, S)$ i.e., let

$$
\bar{T}(\sigma)=0, \quad \bar{S}(\sigma)=\sigma .
$$

Then the solutions (4.29)-(4.32) become uniquely determined functionals of the embeddings via

$$
\begin{aligned}
x^{i}[X](\sigma)= & \frac{1}{2}\left[\bar{x}^{i}\left(X^{+}(\sigma)\right)+\bar{x}^{i}\left(-X^{-}(\sigma)\right)\right. \\
& \left.+\int_{-X^{-}(\sigma)}^{X^{+}(\sigma)} d y \bar{p}^{i}(y)\right],
\end{aligned}
$$

$$
\begin{aligned}
p_{i}[X] & (\sigma) \\
= & \frac{1}{2}\left[X^{+}{ }_{, 1}(\sigma)\left(\bar{x}_{i,+}\left(X^{+}(\sigma)\right)+\bar{p}_{i}\left(X^{+}(\sigma)\right)\right)\right. \\
& +X^{-}{ }_{, 1}(\sigma)\left(\bar{x}_{i,-}\left(-X^{-}(\sigma)\right)\right. \\
& \left.\left.-\bar{p}_{i}\left(-X^{-}(\sigma)\right)\right)\right], \\
\left(x^{-}\right)_{0}= & \left(\overline{x^{-}}\right)_{0}, \\
\left(p_{-}\right)_{0}= & \left(\overline{p_{-}}\right)_{0} .
\end{aligned}
$$

We can, if we like, specify the solutions by incorporating the initial data surface into a (freely specifiable) one-parameter family of embeddings:

$$
\mathrm{X}^{\alpha}=X^{\alpha}(\sigma, \tau), \text { where } X^{\alpha}(\sigma, 0)=\bar{X}^{\alpha}(\sigma) .
$$

A preferred choice is, of course, given by

$$
\begin{aligned}
& T(\sigma, \tau)=\tau, \\
& S(\sigma, \tau)=\sigma .
\end{aligned}
$$

Translating expressions (4.39) and (4.40) back into the original string variables, we find that this choice of foliation corresponds to working in the light-cone gauge. Thus (4.39) becomes

$$
x^{+}=-\left(p_{-}\right)_{0} \tau,
$$

while the derivative of (4.40) yields

$$
p_{-}=\left(p_{-}\right)_{0} \text {. }
$$

The solutions (4.35) and (4.36) then take the familiar form

$$
\begin{aligned}
x^{i}(\sigma, \tau)= & \frac{1}{2}\left(\bar{x}^{i}(\sigma+\tau)+\bar{x}^{i}(\sigma-\tau)\right. \\
& \left.+\int_{\sigma-\tau}^{\sigma+\tau} d y \bar{p}^{i}(y)\right), \quad p_{i}=\dot{x}_{i},
\end{aligned}
$$

while the constraint on the transverse initial data becomes

$$
\int_{S^{\prime}} \bar{p}_{i} \bar{x}_{, 1}^{i}=0 \text {. }
$$

The reduction to the light-cone gauge can be viewed as the final step in a sequence of reductions beginning with the phase space $\Gamma^{\prime}$ of Sec. III A. In this reduction we first eliminate the embeddings by identifying them with the light-cone variables, as we have just described (this can be thought of as gauge fixing). The embedding momenta are then eliminated by solving (3.5). The remaining constraints can be taken as $P_{\alpha} \approx 0$ which, having solved (3.5), become constraints on the string data of exactly the form (4.20) and (4.21). The final step in the reduction amounts to imposing the lightcone gauge conditions (4.39) and (4.40), whereby we fix the allowable embeddings to be leaves of a foliation adapted to 
conformal coordinates. These coordinates are (effectively) defined by the string itself.

Of course, the initial identification of the embedding variables from $\Gamma^{\prime}$ with the string variables is permissible only if the combinations (4.13) and (4.14) [with the identification (4.9) ] truly define spacelike embeddings. This requirement effectively amounts to the validity of the inequality [see (3.17)]

$$
\left(S_{, 1}\right)^{2}-\left(T_{, 1}\right)^{2}>0
$$

As we shall see, the inequality (4.41) is not satisfied precisely when the light-cone gauge fails to be admissible. By admissible we mean that every dynamical trajectory in $\Gamma^{*}$ can be deformed by the induced action of a diffeomorphism on $\boldsymbol{M}$ into a trajectory which satisfies (4.39) and (4.40). Thus consider the equations of motion for the putative embedding variables:

$$
\dot{X}^{ \pm}=\left(N^{1} \pm N\right) X^{ \pm}{ }_{, 1} \text {. }
$$

Representing the diffeomorphism locally as a coordinate transformation, the Jacobian for the transformation from the (almost) global coordinates $(\sigma, \tau)$ to the (conformal) light-cone gauge coordinates is given by

$$
\mathscr{J}=\dot{X}^{+} X^{-}{ }_{, 1}-\dot{X}^{-} X^{+}{ }_{, 1} \text {. }
$$

Using (4.42) we have

$$
\mathscr{J}=2 N X^{+}{ }_{, 1} X^{-}{ }_{, 1} \text {. }
$$

By assumption, $N \neq 0$; thus the coordinate transformation is a good one only when $X^{+}{ }_{, 1} X^{-}{ }_{, 1} \neq 0$. Moreover, given a nonvanishing Jacobian, we can pass to the light-cone gauge only if $X^{+}{ }_{, 1} X^{-}{ }_{, 1}<0$, for if $X^{+}{ }_{, 1} X^{-}{ }_{, 1}>0$ we have

$$
\left(T_{, 1}\right)^{2}-\left(S_{, 1}\right)^{2}>0
$$

Setting $T=\tau$ then leads to a contradiction. Hence the lightcone gauge is admissible only if

$$
X^{+}{ }_{, 1} X^{-}, 1<0 \text {, }
$$

which is just (4.41). It has been noted in the literature ${ }^{12}$ that for the open string, the transformation to light-cone gauge coordinates is singular. We will now indicate how similar difficulties arise (in a somewhat more severe manner) for the closed string which is of interest here. To do this, we should be quite specific on how the closed string phase space is to be defined. As mentioned in Sec. II B, a (slightly generous) definition of $\Gamma$ is the cotangent bundle over the space of smooth embeddings of a circle into $d$-dimensional Minkowski space such that the metric induced on the circle by pulling back the Minkowski metric on $M^{d}$ is positive definite. (Here we ignore the $q, p$ extension of $\Gamma$ as it is irrelevant for the discussion to follow.) This restriction on the string variables translates into

$$
x^{\mu}{ }_{, 1} x_{\mu, 1}>0 \text {. }
$$

Further, at least classically, we can restrict our attention to the constraint surface $\bar{\Gamma} \subset \Gamma$ obtained by imposing (2.47) and (2.48) or equivalently (4.1) and (4.2). These requirements are most transparent when they are written in terms of the $\widetilde{\alpha}^{\mu}, \alpha^{\mu}$ variables introduced previously. Thus $\bar{\Gamma}$ is defined as that subspace of $\Gamma$ that satisfies

$$
\widetilde{\alpha}^{\mu} \widetilde{\alpha}_{\mu}=0
$$

$$
\alpha^{\mu} \alpha_{\mu}=0 \text {. }
$$

The requirements (4.45) are just a restatement of the constraints. Geometrically, (4.45) tell us that at each point of the string (labeled by $\sigma$ ) $\alpha^{\mu}, \widetilde{\alpha}^{\mu}$ are a pair of null vectors in $M^{d}$. Modulo (4.45), (4.44) can be written as

$$
-\alpha^{\mu} \widetilde{\alpha}_{\mu}>0
$$

Equation (4.46) implies that the two null vectors at each $\sigma$ are linearly independent and are given the same orientation in $M^{d}$. Without loss of generality, we can assume that both $\alpha^{\mu}(\sigma)$ and $\widetilde{\alpha}^{\mu}(\sigma)$ are future oriented. Similarly, we will assume that the vectors $k^{\mu}, l^{\mu}$ are future oriented. Now, the admissibility of the light-cone gauge (4.41) translates into

$$
\alpha^{+} \widetilde{\alpha}^{+}>0 \text {. }
$$

Off of $\bar{\Gamma}$, that is, if we only impose (4.44), it is easy to see that (4.47) need not be satisfied. If we restrict attention to $\bar{\Gamma}$, i.e., impose (4.45), it can be shown that the requirements (4.45) and (4.46) are inadequate to guarantee the strict inequality (4.47).

Let us first show that we cannot reverse the inequality in (4.47). To see this, note that since $\alpha^{\mu}$ and $\widetilde{\alpha}^{\mu}$ are future pointing null vectors, they have a negative definite scalar product with the timelike vector $t^{\mu}:=k^{\mu}+l^{\mu}$ :

$$
\widetilde{\alpha}_{\mu} t^{\mu}<0, \quad \alpha_{\mu} t^{\mu}<0 \text {. }
$$

Thus we have

$$
\tilde{\alpha}^{+}+\widetilde{\alpha}^{-}>0, \quad \alpha^{+}+\alpha^{-}>0 .
$$

Furthermore, from (4.45) the products $\alpha^{+} \alpha^{-}$and $\tilde{\alpha}^{+} \widetilde{\alpha}^{-}$ are positive semidefinite. Hence we conclude that the set of functions $\left(\alpha^{ \pm}, \widetilde{\alpha}^{ \pm}\right)$are all greater than or equal to zero; in particular

$$
\widetilde{\alpha}^{+} \alpha^{+} \geqslant 0 \text {. }
$$

Unfortunately, we cannot make the above weak inequality a strong one. A simple example will be sufficient to demonstrate this.

Consider the following point (s) in $\bar{\Gamma}$ :

$$
\begin{aligned}
& \widetilde{\alpha}^{\mu}=\frac{1}{2} k^{\mu}+l^{\mu}+(\cos \sigma) s_{1}^{\mu}+(\sin \sigma) s_{2}^{\mu}, \\
& \alpha^{\mu}=\left(\sin ^{2} \sigma\right) k^{\mu}+l^{\mu}+(\sqrt{2} \sin \sigma) s_{3}^{\mu},
\end{aligned}
$$

where $s_{i}^{\mu}$ are a set of $d-2$ spacelike orthonormal vectors, labeled by $i$, orthogonal to $k^{\mu}$ and $l^{\mu}$. (Note that this example requires $d \geqslant 5$.) These data uniquely determine $p_{\mu}(\sigma)$ and fix $x^{\mu}(\sigma)$ up to an irrelevant additive constant. It is easily verified that this initial data set satisfies (4.45) and (4.46) at each $\sigma$. As for (4.47), we have

$$
\alpha^{+} \tilde{\alpha}^{+}=\frac{1}{2} \sin ^{2} \sigma \geqslant 0 \text {. }
$$

Thus the transformation to the light-cone gauge from this point in phase space is singular at $\sigma=0$. Notice that the intrinsic geometry generated by the above choice of initial data is perfectly regular. If we evolve the data into a string solution using conformal coordinates for $\sigma$ and $\tau$, we find

$$
\begin{aligned}
& \widetilde{\alpha}^{\mu}(\sigma, \tau)=\frac{1}{2} k^{\mu}+l^{\mu}+\cos (\sigma+\tau) s_{1}^{\mu}+\sin (\sigma+\tau) s_{2}^{\mu}, \\
& \alpha^{\mu}(\sigma, \tau)=\sin ^{2}(\sigma-\tau) k^{\mu}+l^{\mu}+\sqrt{2} \sin (\sigma-\tau) s_{3}^{\mu} .
\end{aligned}
$$

The induced metric on $M$ is then given by

$$
d s^{2}=\left(1+2 \sin ^{2}(\sigma-\tau)\right)\left(-d \tau^{2}+d \sigma^{2}\right) .
$$


This metric is completely nonsingular. Moreover, the corresponding curvature vanishes on all of $M$. Thus in terms of the intrinsic geometry, the initial data consist merely of a nonstandard slice of a flat cylinder.

The above example illustrates the fact that strictly speaking, the functions $\mathbf{H}(\vec{V})$ are not globally defined on $\Gamma^{*}$ or even $\bar{\Gamma}$. It may be that the necessary weakening of the inequality in (4.47) is rather harmless: If we work with the constraint surface as defined by (4.7) and (4.8) one can certainly imagine a limiting procedure in which the diffeomorphism Hamiltonians remain defined at the points of $\bar{\Gamma}$ where the light-cone gauge fails. Alternatively, we can simply redefine the phase space such that for a given choice of $k^{\mu}$ and $l^{\mu}$ the inequality (4.47) holds. This is being done implicitly whenever one uses the light-cone gauge in string theory. The redefined phase space, being a dense subset of $\bar{\Gamma}$, may differ insignificantly from $\bar{\Gamma}$ itself, e.g., the light-cone gauge only fails on sets of measure zero in $\bar{\Gamma}$. If this were true, it would seem to imply that the corresponding quantum theory would be unaltered by using the more restricted phase space. However, the inequivalence of light-cone gauge and "covariant" quantization away from the critical dimension suggests otherwise. Clearly, a complete understanding of the difficulties associated with the light-cone gauge will require a separate investigation; we hope to pursue this in a future publication.

To summarize, insofar as the light-cone gauge is admissible, we have succeeded in finding a relatively simple representation of $\operatorname{diff}(M)$ on (a slight extension of) the usual string phase space. Other, more complicated representations can, of course, be contemplated. For example, one could use the component of $x^{\mu}$ along a timelike vector in $M^{d}$ to define the "many-fingered time." The corresponding gauge conditions would certainly be admissible. And it would seem that one could represent $\operatorname{diff}(M)$ globally on $\Gamma$ (or at least $\bar{\Gamma}$ ). We have refrained from following this avenue for pragmatic reasons: The corresponding preferred coordinates are not conformal and one must contend in the quantum theory with unwieldy (square root) operators.

\section{BRST EXTENSIONS}

We hardly need emphasize the central role that the BRST formalism has played in string quantum mechanics and string field theory. More generally, it is currently being revealed ${ }^{13}$ that this way of dealing with constrained systems provides an elegant unification between the classical Hamiltonian structure of such systems and their canonical or path integral methods of quantization. Consequently, it is useful to extend our method of representing $\operatorname{diff}(M)$ to include the phase spaces that are enlarged by the introduction of the ghost variables that feature in the BRST formalism. Our goal in this section will be to obtain BRST extensions of the functions (3.5) and (3.50) and (4.20) and (4.21). Since these "diffeomorphism Hamiltonians" are constrained to vanish, their extended versions should arise as cohomologically trivial functions on the BRST phase space.

Let us begin with the phase space $\Gamma^{\prime}$ obtained through the use of the conformal gauge. The specific form of the BRST charge $\Omega^{\prime}$ that we construct will depend on how we choose to represent the constraint surface $\bar{\Gamma}^{\prime}$. Of course, there will be an infinity of choices, but all the resulting BRST charges can be shown to be related by canonical transformations on the BRST-extended phase space (this is one of the most beautiful features of the classical BRST formalism). For our purposes, it will be most convenient to represent $\bar{\Gamma}$, by

$$
\mathbf{H}_{\alpha}^{\prime} \approx 0
$$

and

$$
\mathscr{H} \approx 0 \approx \mathscr{H}_{1} \text {. }
$$

Local coordinates on the extended phase space, which will be denoted $\hat{\Gamma}^{\prime}$, consist of the string and embedding variables introduced previously along with the Grassmann-valued ghost coordinates $\left(\eta^{\alpha}, \eta, \eta^{1}\right)$ and their respective conjugate momenta $\left(\mathscr{P}_{\alpha}, \mathscr{P}, \mathscr{P}_{1}\right)$. The triplet $\left(\eta^{\alpha}, \eta, \eta^{1}\right)$ consists, respectively, of a pair of scalar functions, a scalar density of weight minus 1 , and a vector (all with respect to $S^{1}$ ). The corresponding conjugate momenta are, respectively, a pair of scalar densities of weight 1 , a density of weight 2 , and a covector density of weight 1 . The only nonvanishing (symmetric) Poisson brackets are

$$
\begin{aligned}
& {\left[\eta^{\alpha}(\sigma), \mathscr{P}_{\beta}\left(\sigma^{\prime}\right)\right]=-\delta_{\beta}^{\alpha} \delta\left(\sigma, \sigma^{\prime}\right),} \\
& {\left[\eta(\sigma), \mathscr{P}\left(\sigma^{\prime}\right)\right]=-\delta\left(\sigma, \sigma^{\prime}\right),} \\
& {\left[\eta^{1}(\sigma), \mathscr{P}_{1}\left(\sigma^{\prime}\right)\right]=-\delta\left(\sigma, \sigma^{\prime}\right) .}
\end{aligned}
$$

To obtain $\Omega^{\prime}$, we will need the Poisson brackets between the constraint functions chosen in (5.1) and (5.2). We have already seen that the functions in (5.1) obey an Abelian algebra, while the functions of (5.2) obey the Dirac algebra (2.54)-(2.56), which is isomorphic to $\operatorname{diff}\left(S^{1}\right) \oplus \operatorname{diff}\left(S^{1}\right)$. The rest of the Poisson brackets are given by

$$
\begin{aligned}
& {\left[\mathbf{H}^{\prime}(\vec{N}), H(M)\right]} \\
& \quad=\int_{S^{\prime}}-\gamma^{-1 / 2} n_{\alpha} N^{\alpha}\left[\left(\partial_{1} M\right) \mathscr{H}_{1}+\partial_{1}\left(M \mathscr{H}_{1}\right)\right] \\
& \quad+\int_{S^{\prime}} X_{\alpha}^{1} N^{\alpha}\left[\left(\partial_{1} M\right) \mathscr{H}+\partial_{1}(M \mathscr{H})\right]
\end{aligned}
$$

and

$$
\begin{aligned}
& {\left[\mathbf{H}^{\prime}(\vec{N}), H\left(M^{1}\right)\right]} \\
& \quad=\int_{S^{\prime}}-\gamma^{-1 / 2} n_{\alpha} N^{\alpha}\left[\left(\partial_{1} M^{1}\right) \mathscr{H}+\partial_{1}\left(M^{1} \mathscr{H}\right)\right] \\
& \quad+\int_{S_{1}} X_{\alpha}^{1} N^{\alpha}\left[\left(\partial_{1} M^{1}\right) \mathscr{H}_{1}+\partial_{1}\left(M^{1} \mathscr{H}_{1}\right)\right] .
\end{aligned}
$$

Relations (5.3) and (5.4) define the remaining first-order structure functions. Notice that these functions are not simply constants, i.e., the Poisson algebra of the constraints (5.1) and (5.2) does not represent a Lie algebra. Hence it is possible that there exist higher-order structure functions. Fortunately, it can be verified by direct computation that these functions can be set to zero. This can be seen without undue labor by noticing that the nontrivial first-order structure functions are functionals of the embeddings only. Using this fact, it is easy to infer from the definition of the secondorder structure functions (B8) that the second-order (and hence all higher-order) structure functions can be set to 
zero. [The crux of the argument is that there is no combination of constraints which involves only $X^{\alpha}(\sigma)$.] The BRST charge then takes the form

$$
\Omega^{\prime}=\mathscr{U}^{\prime}+\int_{S^{\prime}}\left(\eta^{\alpha} \mathbf{H}_{\alpha}^{\prime}+\eta \mathscr{H}+\eta^{1} \mathscr{H}_{1}\right),
$$

where

$$
\begin{aligned}
\mathscr{U}^{\prime}= & \int_{S^{\prime}}\left(\frac{1}{2} \eta-\gamma^{-1 / 2} n_{\alpha} \eta^{\alpha}\right)\left[\left(\partial_{1} \eta\right) \mathscr{P}_{1}+\partial_{1}\left(\eta \mathscr{P}_{1}\right)\right. \\
& \left.+\left(\partial_{1} \eta^{1}\right) \mathscr{P}+\partial_{1}\left(\eta^{1} \mathscr{P}\right)\right] \\
& +\int_{S^{\prime}}\left(\frac{1}{2} \eta^{1}+X_{\alpha}^{1} \eta^{\alpha}\right)\left[\left(\partial_{1} \eta\right) \mathscr{P}+\partial_{1}(\eta \mathscr{P})\right. \\
& \left.+\left(\partial_{1} \eta^{1}\right) \mathscr{P}_{1}+\partial_{1}\left(\eta^{1} \mathscr{P}_{1}\right)\right] .
\end{aligned}
$$

It is straightforward, if somewhat painful, to check that the BRST transformation-the canonical transformation generated by $\Omega^{\prime}$-is a nilpotent transformation on $\hat{\Gamma}^{\prime}$ :

$$
\left[\Omega^{\prime}, \Omega^{\prime}\right]=0 \text {. }
$$

Given the (trivial) observables $\mathbf{H}_{\alpha}^{\prime}$, there is a systematic way of obtaining their BRST invariant extensions (modulo the addition of cohomologically trivial functions). ${ }^{13}$ However, with our choice of $\Omega^{\prime}$, it is easy enough to guess their construction. The cohomologically trivial functions defined by

$$
\hat{\mathbf{H}}_{\alpha}^{\prime}(\sigma):=\left[-\mathscr{P}_{\alpha}(\sigma), \Omega^{\prime}\right]
$$

have all the properties that we desire: They are BRST invariant and equivalent (in the sense of BRST cohomology) to zero. Moreover, by direct calculation or using the results of Appendix B, they satisfy an Abelian Poisson algebra:

$$
\left[\hat{\mathbf{H}}_{\alpha}^{\prime}(\sigma), \hat{\mathbf{H}}_{\beta}^{\prime}\left(\sigma^{\prime}\right)\right]=0 \text {. }
$$

Equation (5.7) is obtained thanks to the Abelian algebra satisfied by $\mathbf{H}_{\alpha}^{\prime}$, from which the cubic ghost terms in $\Omega^{\prime}$ are no more than linear in $\eta^{\alpha}$.

Upon computation of the bracket in (5.6), we find

$$
\hat{\mathbf{H}}_{\alpha}^{\prime}=\mathbf{H}_{\alpha}^{\prime}-\gamma^{-1 / 2} n_{\alpha} \mathscr{H}^{g^{h}}+X_{\alpha}^{1} \mathscr{H}_{1}^{g h},
$$

where

$$
\begin{aligned}
& \mathscr{H}^{g^{h}}=\left(\partial_{1} \eta\right) \mathscr{P}_{1}+\partial_{1}\left(\eta \mathscr{P}_{1}\right)+\left(\partial_{1} \eta^{1}\right) \mathscr{P}+\partial_{1}\left(\eta^{1} \mathscr{P}\right) \\
& \mathscr{H}_{1}^{g h}=\left(\partial_{1} \eta\right) \mathscr{P}+\partial_{1}(\eta \mathscr{P})+\left(\partial_{1} \eta^{1}\right) \mathscr{P}_{1}+\partial_{1}\left(\eta^{1} \mathscr{P}_{1}\right) .
\end{aligned}
$$

From (5.8) we see that $\hat{\mathbf{H}}_{\alpha}^{\prime}$ consists of the original diffeomorphism Hamiltonian plus the ghost corrections $\mathscr{H}^{g h}$ and $\mathscr{H}_{1}^{g h}$, which are generators for the ghost dynamical evolution associated with normal and tangential deformations of a given embedding. As such, the functions (5.9) and (5.10) obey the two-dimensional Dirac algebra. This fact guarantees (5.7) and we again have the homomorphic mapping from $\operatorname{diff}(M)$ into the Poisson algebra of functions on $\widehat{\Gamma}^{\prime}$ given by

$$
\begin{aligned}
& V \rightarrow \vec{V} \rightarrow \hat{\mathbf{H}}^{\prime}(\vec{V}), \\
& {\left[\hat{\mathbf{H}}(\vec{V}), \hat{\mathbf{H}}^{\prime}(\vec{U})\right]=\hat{\mathbf{H}}^{\prime}(-[\vec{V}, \vec{U}]) .}
\end{aligned}
$$

Given a choice of a space-time vector field $\vec{V}$ [or alterna- tively, given a choice of lapse and shift $\left.\left(N, N^{1}\right)\right]$, the Hamiltonian $\hat{\mathbf{H}}^{\prime}(\vec{V})$ [or $\hat{\mathbf{H}}^{\prime}(\vec{N})$ ] generates the appropriate dynamical evolution in $\hat{\Gamma}^{\prime}$. To recover the standard conformal gauge evolution in the BRST-extended phase space, we can choose the foliation to be that given by the auxiliary structure

$$
X^{\alpha}(\sigma, \tau)=\widetilde{Y}^{(\alpha)}(\sigma, \tau),
$$

which implies

$$
N=1, \quad N^{1}=0 .
$$

Any foliation described by this choice of lapse and shift will yield the conventional conformal gauge evolution of the string and ghost variables.

What has been done for $\Gamma^{\prime}$ can also be done for $\Gamma^{\prime \prime}$. The constraint surface is conveniently specified by

$$
\begin{aligned}
& \mathbf{H}_{\alpha}^{\prime \prime} \approx 0, \\
& \mathscr{H} \approx 0 \approx \mathscr{H}_{1}, \\
& \lambda_{(\alpha)} \approx 0 .
\end{aligned}
$$

The first-order structure functions associated with (5.11) and (5.12) are formally identical to $(2.54)-(2.56)$ and (5.3) and (5.4) (with $\mathbf{H}_{\alpha}^{\prime} \rightarrow \mathbf{H}_{\alpha}^{\prime \prime}$ ) except now $\gamma^{-1 / 2} n_{\alpha}$ and $X_{\alpha}^{1}$ are functionals of both $X^{\alpha}(\sigma)$ and $\mu^{(\alpha)}(\sigma)$ via (3.47)(3.49). New structure functions arise from Poisson brackets between $\lambda_{(\alpha)}$ and $\mathbf{H}_{\alpha}^{\prime \prime}$. If we define

$$
\lambda(v):=\int_{S^{\prime}} v^{(a)} \lambda_{(\alpha)},
$$

then

$$
\begin{aligned}
{\left[\lambda(\nu), \mathbf{H}^{\prime \prime}(\vec{M})\right]=} & \int_{S^{\prime}} v^{(\alpha)} M^{\beta}\left[-\gamma^{-1} n_{\beta} n_{\gamma}\left(Y^{-1}\right)_{(\alpha)}^{\gamma}\right. \\
& \times\left(\mathscr{H}+Y^{(\epsilon)}{ }_{, \delta} X^{\delta}{ }_{, 1} \lambda_{(\epsilon), 1}\right) \\
& +\left(Y^{-1}\right)_{(\alpha)}^{\gamma} \gamma^{-1 / 2} n_{\beta} X_{\gamma}^{1}\left(\mathscr{H}_{1}\right. \\
& \left.\left.+\mu^{(\delta)} \lambda_{(\delta), 1}\right)+X_{\beta}^{1} \lambda_{(\alpha), 1}\right]
\end{aligned}
$$

The first-order structure functions include non-constant functions on $\Gamma^{\prime \prime}$ due to the appearance of $\gamma^{-1 / 2} n_{\alpha}, X_{\alpha}^{1}$, $Y_{, \beta}^{(\alpha)}$, and $\left(Y^{-1}\right)_{(\beta)}^{\alpha}$. Nevertheless, as before, the secondorder structure functions can be set to zero. This is essentially because there is no combination of the constraints that is a functional of $X^{\alpha}(\sigma)$ and $\mu^{(\alpha)}(\sigma)$ only.

The BRST charge will be a function on the phase space $\hat{\Gamma}^{\prime \prime}$, which has local coordinates given by those chosen earlier for $\hat{\Gamma}^{\prime}$ along with $\left(\lambda_{(\alpha)}, \mu^{(\beta)}\right)$ and the additional ghosts $\left(\eta^{(\alpha)}, \mathscr{P}_{(\beta)}\right)$. The ghost $\eta^{(\alpha)}$ is a pair of (Grassman valued) scalar densities of weight 1 , while its conjugate momentum $\mathscr{P}_{(\beta)}$ consists of two scalars: They satisfy the Poisson brackets

$$
\left[\eta^{(\alpha)}(\sigma), \mathscr{P}_{(\beta)}\left(\sigma^{\prime}\right)\right]=-\delta_{(\beta)}^{(\alpha)} \delta\left(\sigma, \sigma^{\prime}\right)
$$

In these coordinates $\Omega^{\prime \prime}$ takes the form

$$
\begin{aligned}
\Omega^{\prime \prime}= & \mathscr{U}^{\prime \prime}+\mathscr{U}^{\prime}+\int_{S^{\prime}}\left(\eta^{\alpha} \mathbf{H}_{\alpha}^{\prime \prime}\right. \\
& \left.+\eta \mathscr{H}+\eta^{\prime} \mathscr{H}_{1}+\eta^{(\alpha)} \lambda_{(\alpha)}\right),
\end{aligned}
$$


where

$$
\begin{aligned}
\mathscr{U}^{\prime \prime}= & \int_{S^{\prime}} \eta^{(\alpha)} \eta^{\beta}\left[-\gamma^{-1} n_{\beta} n_{\gamma}\left(Y^{-1}\right)_{(\alpha)}^{\gamma}\right. \\
& \times\left(\mathscr{P}+Y^{(\epsilon)}{ }_{, \delta} X_{, 1}^{\delta} \mathscr{P}_{(\epsilon), 1}\right) \\
& +\left(Y^{-1}\right)_{(\alpha)}^{\gamma} \gamma^{-1 / 2} n_{\beta} X_{\gamma}^{1}\left(\mathscr{P}_{1}+\mu^{(\delta)} \mathscr{P}_{(\delta), 1}\right) \\
& \left.+X_{\beta}^{1} \mathscr{P}_{(\alpha), 1)}\right] .
\end{aligned}
$$

It is important to keep in mind that in (5.15) $\mathscr{U}^{\prime}$ ' is given by (5.5b), but $n_{\alpha}$ and $X_{\alpha}^{1}$ are to be thought of as functionals of $X^{\alpha}(\sigma)$ and $\mu^{(\alpha)}(\sigma)$ via (3.47)-(3.49).

As before, a BRST invariant extension of $\mathbf{H}_{\alpha}^{\prime \prime}$ is obtained from the following Poisson bracket:

$$
\begin{aligned}
\hat{\mathbf{H}}_{\alpha}^{\prime \prime}(\sigma):= & {\left[-\mathscr{P}_{\alpha}(\sigma), \Omega^{\prime \prime}\right]=\mathbf{H}_{\alpha}^{\prime \prime}(\sigma) } \\
& -\gamma^{-1 / 2} n_{\alpha} \mathscr{H}^{g h}+X_{\alpha}^{1} \mathscr{H}_{1}^{g h}+V_{\alpha}^{\prime \prime},
\end{aligned}
$$

where

$$
\begin{aligned}
V_{\alpha}^{\prime \prime}= & -\gamma^{-1 / 2} n_{\alpha} \eta^{(\beta)}\left[-\gamma^{-1 / 2} n_{\gamma}\left(Y^{-1}\right)_{(\beta)}^{\gamma}\right. \\
& \times\left(\mathscr{P}+Y_{, \delta}^{(\epsilon)} X_{, 1}^{\delta} \mathscr{P}_{(\epsilon), 1}\right) \\
& \left.+\left(Y^{-1}\right)_{(\beta)}^{\gamma} X_{\gamma}^{1}\left(\mathscr{P}_{1}+\mu^{(\delta)} \mathscr{P}_{(\delta), 1}\right)\right] \\
& +X_{\alpha}^{1} \eta^{(\beta)} \mathscr{P}_{(\beta), 1} .
\end{aligned}
$$

Once again, by virtue of the Abelian Poisson bracket algebra obeyed by $\mathbf{H}_{\alpha}^{\prime \prime}$, we have

$$
\left[\hat{\mathbf{H}}_{a}^{\prime \prime}(\sigma), \hat{\mathbf{H}}_{\beta}^{\prime \prime}\left(\sigma^{\prime}\right)\right]=0 .
$$

The mapping

$$
V \rightarrow \vec{V} \rightarrow \hat{\mathbf{H}}^{\prime \prime}(\vec{V})
$$

then serves to represent $\operatorname{diff}(M)$ in terms of cohomologically trivial observables on $\widehat{\Gamma}^{\prime \prime}$.

Finally, we turn to the phase space $\Gamma^{*}$ of Sec. IV. The constraints on this phase space consist of the vanishing of the diffeomorphism Hamiltonians (4.20) and (4.21):

$$
\mathbf{H}_{\alpha} \approx 0 \text {, }
$$

along with the "shift of origin" constraint (4.34):

$$
R:=\int_{S^{\prime}}\left(\left(X^{+}{ }_{, 1}\right)^{-1} \tilde{\alpha}_{i} \tilde{\alpha}^{i}+\left(X^{-}{ }_{, 1}\right)^{-1} \alpha_{i} \alpha^{i}\right) \approx 0 .
$$

These constraint functions satisfy an Abelian Poisson bracket algebra; hence the BRST charge takes its simplest possible form. In terms of canonical variables on $\hat{\Gamma}^{*}$ consisting of the string variables of Sec. IV and the ghost canonical pairs $\left(\eta^{\alpha}, \mathscr{P}_{\alpha}\right),\left(\eta_{0}, \mathscr{P}_{0}\right)$, where the latter variables are constants on $S^{1}$, we have

$$
\Omega=\eta_{0} R+\int_{S^{\prime}} \eta^{\alpha} \mathbf{H}_{\alpha}
$$

The BRST extensions of the constraint functions are just the functions themselves:

$$
\begin{aligned}
& \hat{\mathbf{H}}_{\alpha}=\left[-\mathscr{P}_{\alpha}, \Omega\right]=\mathbf{H}_{\alpha}, \\
& \hat{R}=\left[-\mathscr{P}_{0}, \Omega\right]=R .
\end{aligned}
$$

We see that the representation of $\operatorname{diff}(M)$ on $\hat{\Gamma}^{\star}$ is a trivial extension of what was done in Sec. IV for $\Gamma^{*}$.

The investigations of McMullan and Paterson ${ }^{14}$ have revealed that the Abelian constraint functions play an im- portant role in arriving at consistent factor orderings for the non-Abelian (i.e., projected) constraint functions by using the BRST formalism in the quantum theory. It is therefore of interest to relate $\Omega$ defined above to the BRST charge $\widetilde{\Omega}$ associated with the projected constraints:

$$
\widetilde{\Omega}=\mathscr{U}+\eta_{0} R+\int_{S^{\prime}}\left(\eta \mathscr{H}+\eta^{1} \mathscr{H}_{1}\right)
$$

where

$$
\begin{aligned}
\mathscr{U}= & \int_{S^{1}}\left[\eta\left(\left(\partial_{1} \eta\right) \mathscr{P}_{1}+\left(\partial_{1} \eta^{1}\right) \mathscr{P}\right)\right. \\
& \left.+\eta^{1}\left(\left(\partial_{1} \eta^{1}\right) \mathscr{P}_{1}+\left(\partial_{1} \eta\right) \mathscr{P}\right)\right]
\end{aligned}
$$

The charges $\Omega$ and $\widetilde{\Omega}$ are related by a canonical transformation on $\hat{\Gamma}^{*}$. Define

$$
\eta^{( \pm)}=\left(\eta \pm \eta^{1}\right), \quad \mathscr{P}_{( \pm)}=\frac{1}{2}\left(\mathscr{P} \pm \mathscr{P}_{1}\right)
$$

the canonical transformation is obtained by first performing (4.13) $-(4.18)$ and then making the transformation

$$
\begin{aligned}
& \eta^{ \pm}=\left(X^{ \pm}{ }_{, 1}\right) \eta^{( \pm)} \\
& \mathscr{P}_{ \pm}=\left(X_{{ }_{1}}\right)^{-1} \mathscr{P}_{( \pm)}, \\
& P_{ \pm}=\widetilde{P}_{ \pm}+\partial_{1}\left[\left(X_{{ }_{11}}\right)^{-1} \eta^{( \pm)} \mathscr{P}_{( \pm)}\right]
\end{aligned}
$$

along with the identity transformation on the remaining variables.

In contrast to gauge theories, where constraints are linear in momenta, this transformation is not a point transformation; it necessarily mixes the original string coordinates and momenta through the functionals $X^{ \pm}(\sigma)$. Note, however, that once the transformation (4.13)-(4.18) has been made, the subsequent Abelianizing transformation (5.24)(5.26) is a point transformation on $\hat{\Gamma}^{*}$. In other words, in a manifestly parametrized theory, the transformation from the projected constraint functions to the unprojected constraint functions is a point canonical transformation in the BRST phase space.

\section{THE ROLE OF THE CONFORMAL GROUP}

We have now exhibited several string phase space representations of the Lie algebra $\operatorname{diff}(M)$. However, as pointed out in Sec. II, we cannot expect to be able to "exponentiate" the corresponding infinitesimal canonical transformations to yield a canonical representation of the full group $\operatorname{Diff}(M)$. The difficulty stems from the way in which the diffeomorphisms act on the spacelike embeddings [as in (2.28)] to produce, in general, nonspacelike embeddings.

One is naturally led to ask if there exist interesting subgroups of $\operatorname{Diff}(M)$ which do preserve the spacelike character of the embeddings. Generalizing for a moment to an $n$-dimensional manifold $M^{n}$ with Cauchy surface $\Sigma$, the answer is essentially negative. It is true that if a metric $g_{a b}$ on $M^{n}$ admits Killing vectors, then the corresponding conserved charges do generate a subgroup which is representable on $E m b_{g}\left(\Sigma, M^{n}\right)$. To see this, consider two embeddings related by $\phi \in \operatorname{Diff}\left(M^{n}\right)$ :

$$
X: \Sigma \rightarrow M^{n}, \quad \bar{X}: \Sigma \rightarrow M^{n}, \quad \bar{X}=\phi \circ X .
$$


If the original embedding is spacelike, the induced metric

$$
\gamma=X^{*} g
$$

is positive definite. The metric induced on the new embedding is obtained via

$$
\bar{\gamma}=\bar{X} * g=(\phi \circ X)^{*} g=X^{*}\left(\phi^{*} g\right)
$$

If $\phi$ is generated by a Killing vector, then

$$
\phi^{*} g=g
$$

and the new embedding is also spacelike. In fact, we see that all we really need is for $\phi$ to be a conformal isometry since then

$$
\phi^{*} g=e^{2 \Psi} g
$$

for some nonvanishing function $\Psi: M^{n} \rightarrow R$. In this case the dynamical field of interest must have a Weyl invariant action functional in order to produce the appropriate conserved charge, which then serves to represent the conformal group of isometries.

For a generic space-time $\left(M^{n}, g\right)$ there are no conformal isometries or at best, a finite-dimensional group of them. In our two-dimensional cylindrical space-time the situation is much more interesting. Indeed, the dimension and topology of $M=R \times S^{1}$ admit an infinite-dimensional group of conformal isometries irrespective of the metric (see Appendix A). The phase space of the Weyl invariant string will then carry a representation of this rather large group. As we have already seen, the conformal group serves as a symmetry group for the string. What is a bit more surprising is that this group can also play the role of a dynamical group. If $\operatorname{Conf}(M, g)$ is to allow this reinterpretation, we must show that any two spacelike embeddings can be linked by the action of a conformal isometry. This result, implicitly contained in the fact that the two-dimensional Dirac algebra is isomorphic to $\operatorname{conf}(M, g)$, can be directly demonstrated as follows.

Consider two spacelike embeddings related by a diffeomorphism as in (6.1). We will show that there exists a conformal isometry $\psi \in \operatorname{Conf}(M, g)$ such that

$$
\bar{X}=\psi \circ X
$$

The proof is by construction. Introduce a conformal coordinate system $\mathrm{X}^{ \pm}$on $\boldsymbol{M}$ :

$$
d s^{2}=-e^{2 \omega(X)} d \mathrm{X}^{+} d \mathrm{X}^{-}
$$

for a manifold homeomorphic to $R^{2}$ this is a global coordinate chart. For our cylindrical space-time some care is needed at the points that are identified to produce circles. As summarized in Appendix A, within this chart conformal isometries are characterized by a pair of one-dimensional diffeomorphisms

$$
\psi^{ \pm}=\psi^{ \pm}\left(\mathrm{X}^{ \pm}\right)
$$

[in the notation of Appendix A this is case (i); case (ii) is handled similarly]. Next, we use the fact that the null geodesics $\mathrm{X}^{ \pm}=$const intersect each spacelike embedding (Cauchy surface) once and only once, so that both $\mathrm{X}^{+}$and $\mathrm{X}^{-}$serve as good coordinates on the embedding. In particular, the functions $\mathrm{X}^{ \pm}=X^{ \pm}(\sigma)$ are invertible, i.e., we can form

$$
\sigma=\sigma^{+}\left(\mathrm{X}^{+}\right)
$$

or

$$
\sigma=\sigma^{-}\left(\mathrm{X}^{-}\right)
$$

Given any $\phi \in \operatorname{Diff}(M)$ that preserves the spacelike character of an embedding, we construct the corresponding $\psi \in \operatorname{Conf}(M, g)$ via

$$
\psi^{ \pm}\left(\mathrm{X}^{ \pm}\right)=\phi^{ \pm}\left(\sigma\left(\mathrm{X}^{ \pm}\right)\right)
$$

where

$$
\phi(\sigma)=\phi(X(\sigma))
$$

That the conformal isometry (6.4) does the job (6.2) is guaranteed by the identity

$$
\begin{aligned}
\bar{X} \pm(\sigma)=\psi^{ \pm}\left(X^{ \pm}(\sigma)\right) & =\phi^{ \pm}(\sigma X \pm(\sigma)) \\
& =\phi^{ \pm}(\sigma)=\bar{X} \pm(\sigma) .
\end{aligned}
$$

Thus in two dimensions, the symmetry group Conf $(M, g)$ can in fact also be taken as the dynamical group. That this is a somewhat remarkable occurrence can be seen by comparing the present situation with that arising in canonical geometrodynamics: There, the Poisson algebra of constraints ("hypersurface deformation algebra") is not a true Lie algebra. With some labor (analogous to what was done in Sec. III) one can extract a realization of the dynamical algebra $\operatorname{diff}(M)$, but no subgroup of $\operatorname{Diff}(M)$ exists which can play the role of the dynamical group. In string theory, the hypersurface deformation algebra (2.54)(2.56) is a true Lie algebra isomorphic to conf $(M, g)$ and, as we have just seen, is associated with a group representation on the string phase space. It has, of course, been known for some time (and from various perspectives) that the conformal group plays a fundamental role in string theory. We now see that from the perspective of hypersurface dynamics, $\operatorname{Conf}(M, g)$ is a valid substitute for the would-be dynamical group Diff $(M)$.

\section{DISCUSSION}

The multiplicity of ways that we have uncovered to represent $\operatorname{diff}(M)$ for the canonical string each has its own advantages and disadvantages. It is amusing to note that all the ways of making the canonical string manifestly covariant rest on the validity of associated gauge choices, in particular the conformal, harmonic, and light-cone gauges. Using the conformal and harmonic gauges we were able to construct $\operatorname{diff}(\boldsymbol{M})$ comoments which were globally defined on the respective extended phase spaces $\Gamma^{\prime}$ and $\Gamma^{\prime \prime}$. Moreover, manifest (target space) Poincaré covariance is maintained throughout. The price we pay is that we must extend the usual string phase space. Whether or not these extended phase spaces have a useful role in string theory remains to be seen. We should point out that $\Gamma^{\prime}$ has featured in one approach to string field theory. ${ }^{15}$ We have not investigated whether a corresponding approach using $\Gamma^{\prime \prime}$ is viable. From the gravitational perspective, $\Gamma^{\prime}$ is analogous to the extended phase space based on the Gaussian coordinate conditions used in Ref. 2. The space $\Gamma^{\prime \prime}$ serves as a useful model for the generalization of the techniques of Ref. 2 to the harmonic 
gauge in general relativity. Here the string is serving its purpose as a gravitational paradigm quite well: The techniques developed in Sec. III B have a straightforward generalization to geometrodynamics in the harmonic gauge. ${ }^{16} \mathrm{We}$ are thus able to tie up a loose end left dangling in Ref. 2.

When we try to represent $\operatorname{diff}(M)$ on the usual string phase space $\Gamma$, a fairly large price must be paid. First, strictly speaking, the use of an enlarged phase space $\left(\Gamma^{*}\right)$ is necessary owing to the "shift of origin" symmetry which remains after passing to the light-cone gauge (this problem is absent for the open string). The definition of the embedding momentum $P_{S}$ that accompanies the enlargement is somewhat cumbersome: From (4.16) we see that $P_{S}$ is a distribution (off the constraint surface). Ultimately, this feature of the formalism has its origin in our use of local coordinates on the circle. A global, coordinate-independent treatment would make the extraction of the embedding phase space a bit more elegant. We will present this improvement elsewhere. A more serious drawback of using $\Gamma^{*}$ to represent $\operatorname{diff}(M)$ is that the diffeomorphism Hamiltonians are not globally defined on $\Gamma^{*}$. As was shown, this difficulty is intimately associated with the failure of the light-cone gauge. Finally, it is necessary to break manifest Lorentz invariance in order to extract the embedding phase space from $\Gamma^{*}$. Classically this is of no real consequence. By translating the usual Poincaré group generators using $(4.13)-(4.18)$, one can show that the Lie algebra is still realized. A corresponding statement may not survive quantization, as will be discussed below.

A preliminary investigation of the quantum mechanical representation of $\operatorname{diff}(M)$ is given in Ref. 5. Let us summarize here the gist of this work. The strategy of Ref. 5 is to use the fact that, as shown here, the canonical string can be explicitly reformulated as a parametrized field theory. Quantization of parametrized scalar fields on cylindrical space-times has been extensively studied in Ref. 17. Using the techniques developed there, we have reached the following results. The quantization based on $\Gamma^{\prime}$ does allow for an anomaly-free representation of $\operatorname{diff}(M)$ for any value of the target space dimension $d$. Thus it is consistent to select physical states by requiring that they be annihilated by the (suitably ordered) quantum mechanical diffeomorphism Hamiltonians. However, one still must impose the original constraints (2.47) and (2.48). To do this consistently, one must therefore also represent the conformal symmetry group on the string Hilbert space. This can only be done projectively. Thus within the canonical framework we recover the results of Polya$\operatorname{kov}^{3}: \operatorname{diff}(M)$ covariance is maintained at the expense of conformal symmetry. As expected, by incorporating the BRST ghosts, according to Sec. V, Conf $(M, g)$ can be represented without anomaly in the critical dimension $d=26$. The quantization based on $\Gamma^{\prime \prime}$ has not been followed in Ref. 5.

When we study the quantization based on $\Gamma^{*}$, we find again that $\operatorname{diff}(M)$ can be represented without anomaly irrespective of the value of $d$. Since the diffeomorphism Hamiltonians are now just combinations of the original constraint functions (2.47) and (2.48) [modulo (4.11)], this means that a true "Dirac quantization" is possible within this framework. The symmetry group $\operatorname{Conf}(M, g)$ is still projec- tively represented (now only for the transverse variables), but this is of no consequence for quantization $a$ la Dirac. Of course, it is necessary to sacrifice manifest Lorentz invariance to achieve this result. If it could be shown that Lorentz invariance is still present, we would have a new, nontrivial quantization of the string away from the critical dimension. (Of course, we do not maintain that this quantization needs to be useful from the point of view of elementary particle physics.) From the quantum gravity perspective, the role of the Lorentz group in the quantization based on $\Gamma^{*}$ is equally interesting. The quantization procedure that allows $\operatorname{diff}(M)$ to be represented without anomaly necessarily treats the kinematic variables, i.e., the embeddings, quite differently from the dynamical variables, i.e., the transverse variables [modulo (4.11)]. The Lorentz group, if it does act in the quantum theory, mixes the kinematical "many-fingered time" variables with the dynamical fields. Thus we have, in a rather simple setting, a good model for exploring the role of time in quantum gravity. At this writing it is unknown whether the Lorentz group is represented in the critical dimension or otherwise. This issue will be investigated in a future publication.

\section{ACKNOWLEDGMENT}

This work was supported in part by NSF Grant No. PHY 8503653 to the University of Utah.

\section{APPENDIX A: THE TWO-DIMENSIONAL CONFORMAL GROUP}

Here we present some needed results from two-dimensional (Lorentzian) geometry as applied to $M=R \times S^{\prime}$. Here $M$ can be defined as the Cartesian product of $(-\infty, \infty)$ and $[0,2 \pi]$, with the points 0 and $2 \pi$ identified:

$$
\boldsymbol{M}=(-\infty, \infty) \times[0,2 \pi] \text {. }
$$

Coordinates $X^{0}=T \in(-\infty, \infty)$ and $X^{1}=S \in[0,2 \pi]$ will be referred to as "standard coordinates" on $\boldsymbol{M}$.

As is well known, because the Euler number of $M$ is zero, every metric on $M$ is conformal to a flat metric:

$$
g_{a b}=e^{2 \omega} \eta_{a b}, \quad R(\eta)=0 .
$$

Here $\omega$ is a function on $M$. Without further assumptions, neither $\omega$ nor $\eta_{a b}$ is unique. Given a choice for $\omega$ and $\eta_{a b}$, we can introduce conformal coordinates, i.e., standard coordinates on $M$ such that the line element takes the form

$$
d s^{2}=e^{2 \omega(X)}\left(-d \mathrm{~T}^{2}+d \mathrm{~S}^{2}\right) .
$$

Alternatively, we can introduce null coordinates $\mathbf{X}^{ \pm}=\mathbf{T} \pm \mathbf{S}$ such that

$$
d s^{2}=-e^{2 \omega(X)} d \mathrm{X}^{+} d \mathrm{X}^{-} \text {. }
$$

The curves $\mathrm{X}^{+}=$const and $\mathrm{X}^{-}=$const are null geodesics. (Every null curve in two dimensions is a geodesic.) Nonaffine (in general) parameters for $\mathrm{X}^{ \pm}=$const are provided by $\mathrm{X}^{\mp}$.

The group $\operatorname{Conf}(M, g)$ of conformal isometries is that subgroup of the diffeomorphism group $\operatorname{Diff}(M)$ which has the effect of rescaling the metric by a function. More precisely, if $\psi \in \operatorname{Conf}(M, g)$, then 


$$
\left(\psi^{*} g\right)_{a b}=e^{2 \Psi} g_{a b}
$$

for some $\Psi: M \rightarrow R$. Clearly, $\psi \in \operatorname{Conf}(M, g)$ must also serve as a conformal isometry for the conformally related flat metric, i.e., $\psi \in \operatorname{Conf}(\boldsymbol{M}, \boldsymbol{\eta})$. A coordinate expression $\psi^{\alpha}$ for $\psi$ is most easily obtained in terms of the null coordinates $\mathrm{X}^{ \pm}$. The condition (A2) for the metric (A1) becomes

$$
\begin{aligned}
& \psi^{+}, \psi^{-},+=0 \text {, } \\
& \psi^{+},-\psi^{-},-,=0 \text {, } \\
& \psi^{+},{ }_{,} \psi^{-},-+\psi^{+},-\psi^{-},{ }_{,}=e^{2 \boldsymbol{\Psi}(X)} \text {. }
\end{aligned}
$$

The transformation is nonsingular provided that

$$
J=\psi^{+},{ }_{+} \psi^{-},--\psi^{+}, \psi^{-},+\neq 0 .
$$

From these requirements we have either

$$
\text { (i) } \psi^{ \pm}=\psi^{ \pm}\left(X^{ \pm}\right), \quad \psi^{+}, \psi^{-}, \psi^{-}>0 \quad\left(J_{>} 0\right)
$$

or

(ii) $\psi^{ \pm}=\psi^{ \pm}\left(X^{\mp}\right), \quad \psi^{+}, \psi^{-},{ }_{+}>0 \quad(J<0)$.

Infinitesimal transformations in $\operatorname{Conf}(M, g)$ are generated by conformal Killing vectors which, as usual, form the corresponding Lie algebra conf $(M, g)$. In the null coordinates introduced above, the conformal Killing equation

$$
\mathscr{L}{ } g_{a b}=2 \alpha(\mathrm{X}) g_{a b}
$$

implies

$$
v^{+},-=0=v^{-},+
$$

Thus $v^{ \pm}=v^{ \pm}\left(X^{ \pm}\right)$and we conclude that $\operatorname{Conf}(M, g)$ is disconnected: Type (i) transformations are connected to the identity and the corresponding Lie algebra is isomorphic to $\operatorname{diff}\left(S^{1}\right) \oplus \operatorname{diff}\left(S^{1}\right)$. Type (ii) transformations are not connected to the identity.

Finally, we note that a conformal isometry is completely determined by its action on the Cauchy surfaces $\mathrm{T}=$ const in $M$. This is obvious from the coordinate forms we have presented for $\psi$; given $\psi^{ \pm}(\mathrm{S})$, type (i) transformations are determined by

$$
\psi^{ \pm}=\psi^{ \pm}\left(\mathrm{S}=\mathrm{X}^{ \pm}\right)
$$

and type (ii) transformations are given by

$$
\psi^{ \pm}=\psi^{ \pm}\left(\mathrm{S}=\mathrm{X}^{\mp}\right) \text {. }
$$

This result can be generalized to any Cauchy surface

$$
\mathrm{X}^{ \pm}=X^{ \pm}(\sigma)
$$

by using the techniques of Sec. VI. Thus given the restriction of a conformal isometry to the circle (A6), i.e., given $\psi^{ \pm}(\sigma)$, we invert the functions in (A6) and obtain the form of $\psi$ valid on all $M$ :

$$
\psi^{ \pm}\left(\mathrm{X}^{ \pm}\right)=\psi^{ \pm}\left(\sigma\left(\mathrm{X}^{ \pm}\right)\right)
$$

or

$$
\psi^{ \pm}\left(\mathrm{X}^{\mp}\right)=\psi^{ \pm}\left(\sigma\left(\mathrm{X}^{\mp}\right)\right) .
$$

\section{APPENDIX B: BACKGROUND MATERIAL ON THE BRST FORMALISM}

In this Appendix we give background material on the BRST formalism needed for Sec. V. The Hamiltonian approach to BRST results from the work of Batalin et al. ${ }^{18}$ The formalism is described in considerable detail by Henneaux ${ }^{13}$ and for the most part we will follow his notation and conventions. For the purpose of this Appendix it will be necessary to suspend the notation developed in the main part of the present paper.

At the classical level, the BRST formalism is a mathematically elegant way of capturing the additional structure that arises for Hamiltonian systems when one introduces first class constraints. ${ }^{1}$ Recall that the basic ingredients of such systems consist of a phase space $\Gamma$ and a constraint surface $\bar{\Gamma} \subset \Gamma$ which can be specified (locally) by the vanishing of a set of functions on $\Gamma$ :

$$
\phi_{\alpha}=0
$$

these functions satisfy a closed Poisson bracket algebra

$$
\left[\phi_{\alpha}, \phi_{\beta}\right]=C_{\alpha \beta}^{\gamma} \phi_{\gamma},
$$

where in general, $C_{\alpha \beta}^{\gamma}$ are nonconstant functions on $\Gamma$. All physical dynamics take place on $\bar{\Gamma}$. However, as a result of (B2) the Hamiltonian vector fields associated with $\phi_{\alpha}$ are both tangent and normal to $\bar{\Gamma}$; hence $\bar{\Gamma}$ cannot be a symplectic submanifold of $\Gamma$. The physical symplectic manifold can be defined by a complete set of "observables," which are functions on $\Gamma$ which are weakly "gauge invariant" in the sense that the variation of the observables associated with the infinitesimal canonical transformations generated by the constraint functions vanishes upon restriction to $\bar{\Gamma}$. Thus if $A: \Gamma \rightarrow R$ is an observable, we have

$$
\left[A, \phi_{\alpha}\right]=\Lambda_{\alpha}^{\beta} \phi_{\beta}
$$

for some functions $\Lambda_{\alpha}^{\beta}$. Any two observables that differ by a combination of constraint functions are considered equivalent:

$$
A \sim A+\Lambda^{\alpha} \phi_{\alpha},
$$

where $\Lambda^{\alpha}$ are functions on $\Gamma$. In particular, the functions $\phi_{\alpha}$ are equivalent to zero. In gauge theories the observables are simply the (weakly) gauge invariant functions. In parametrized theories, which are of central interest here, the "observables" can be identified with the constants of motion.

All the pertinent information contained in the constraints (e.g., the constraint algebra, etc.) can be neatly stored in a single function on an extended phase space $\hat{\Gamma} \supset \Gamma$; this function is the BRST charge $\Omega$. Here $\hat{\Gamma}$ can be obtained by introducing the Grassmann-valued "ghosts" $\eta^{\alpha}$ and their conjugate momenta $\mathscr{P}_{\alpha}$. The only nonvanishing Poisson brackets involving these new variables are

$$
\left[\eta^{\alpha}, \mathscr{P}_{\beta}\right]=-\delta_{\beta}^{\alpha} \text {. }
$$

Brackets between two Grassmann-odd functions on $\hat{\Gamma}$ are symmetric; otherwise, the Poisson brackets are, as usual, antisymmetric in their arguments. The BRST charge can be specified by two conditions:

$$
\begin{aligned}
& \Omega\left(\mathscr{P}_{\alpha}=0\right)=\eta^{\alpha} \phi_{\alpha}, \\
& {[\Omega, \Omega]=0 .}
\end{aligned}
$$

The requirement (B5) simply informs $\Omega$ about the existence of $\bar{\Gamma}$. The requirement (B6) then summarizes the algebra (B2), as well as all the identities that arise by taking repeated Poisson brackets of (B2) with the constraint functions and 
using the Jacobi identity. The solution of (B5) and (B6) is given by ${ }^{13}$

$$
\Omega=\sum_{n=0}^{R} U_{\alpha_{n} \cdots \alpha_{n}}^{\beta_{1} \cdots \beta_{n}} \eta^{\alpha_{n} \cdots} \eta^{\alpha_{n} \mathscr{P}_{\beta_{n}}} \cdots \mathscr{P}_{\beta_{1}} .
$$

The integer $R$ is known as the rank of the constraint functions $\phi_{\alpha}$. The coefficients $U$ are functions on $\Gamma$ and are known as the structure functions for the constraint algebra. In particular, we have the zeroth-order structure functions

$$
U_{\alpha}=\phi_{\alpha}
$$

and the first-order structure functions

$$
U_{\alpha \beta}^{\gamma}=-\frac{1}{2} C_{\alpha \beta}^{\gamma} \text {. }
$$

The general expressions for the higher-order structure functions are given in Ref. 13. We will only need to know the definition of the second-order structure functions. They can be defined via

$$
2 U_{\alpha \beta \gamma}^{\delta \epsilon} \phi_{\epsilon}=D_{[\alpha \beta \gamma]}^{\delta},
$$

where

$$
D_{\alpha \beta \gamma}^{\delta}=\left[U_{\alpha \beta}^{\delta}, \phi_{\gamma}\right]+2 U_{\alpha \beta}^{\kappa} U_{\gamma \kappa}^{\delta} .
$$

All BRST charges constructed in this paper are of rank 1. This means that the second-order-and all higher-orderstructure functions can be set to zero. Note that for the rank to be 1 it is sufficient, but not necessary that the first-order structure functions are constants on $\Gamma$.

It can be shown ${ }^{13}$ that any observable $A$ defined by (B3) and (B4) can be extended to a function $\hat{A}$ on $\hat{\Gamma}$ which is BRST invariant, i.e., invariant under the nilpotent canonical transformation generated by $\Omega$ :

$$
[\hat{A}, \Omega]=0 .
$$

The extension of $A$ is unique up to the addition of terms of the form

$$
B=[\Lambda, \Omega],
$$

where $\Lambda$ is a function on $\hat{\Gamma}$. Thus it is natural to define BRST observables as equivalence classes of functions which are BRST invariant; two functions belong to the same equivalence class if they differ by a "cohomologically trivial" function of the form (B10):

$$
\hat{A} \sim \hat{A}+[\Lambda, \Omega] \text {. }
$$

The cohomologically trivial functions are observables, but they are equivalent to zero. Note that (B9) is the BRST generalization of (B3) and (B11) is the generalization of the equivalence relation (B4).

In Sec. V we are interested in computing the BRST extensions of the diffeomorphism Hamiltonians which, being constraint functions, are trivial observables in the usual framework. Their extension should likewise be trivial, i.e., of the form (B10). The key feature of the BRST-extended diffeomorphism Hamiltonians is that they take the form of the original diffeomorphism Hamiltonians plus ghost representatives of $\operatorname{diff}(M)$. The extended functions must still satisfy an Abelian Poisson bracket algebra. To see how this comes about, consider partitioning the constraints $\phi_{\alpha}$ as

$$
\phi_{\alpha}=\left(H_{A}, \chi_{a}\right),
$$

where $H_{A}$ are to play the role of the unextended diffeomor- phism Hamiltonians and $\chi_{a}$ are any remaining constraint functions. Assuming $\phi_{\alpha}$ are of rank 1, we have

$$
\Omega=\eta^{\alpha} \phi_{\alpha}+U_{\alpha \beta}^{\gamma} \eta^{\beta} \eta^{\alpha} \mathscr{P}_{\gamma}
$$

The BRST extension of $H_{A}$ is obtained via

$$
\hat{H}_{A}=-\left[\mathscr{P}_{A}, \Omega\right]=H_{A}-2 U_{A \beta}^{\gamma} \eta^{\beta} \mathscr{P}_{\gamma} .
$$

We are interested in computing the Poisson brackets between $\hat{H}_{A}$. Using the (graded) Jacobi identity we find

$$
\begin{aligned}
{\left[\hat{H}_{A}, \hat{H}_{B}\right]=} & {\left[\mathscr{P}_{B},\left[\Omega, \hat{H}_{A}\right]\right]-\left[\Omega,\left[\hat{H}_{A}, \mathscr{P}_{B}\right]\right] } \\
= & 2 U_{A B}^{\gamma} \hat{\phi}_{\gamma}+2 \mathscr{P}_{\gamma}\left[\Omega, U_{A B}^{\gamma}\right] \\
& +\left[\mathscr{P}_{B},\left[\Omega, \hat{H}_{A}\right]\right],
\end{aligned}
$$

where

$$
\hat{\phi}_{\gamma}:=-\left[\mathscr{P}_{\gamma}, \Omega\right] .
$$

The third term in (B13) can be eliminated, since $\widehat{H}_{A}$ is cohomologically trivial, by using the Jacobi identity

$$
\left[\Omega, \hat{H}_{A}\right]=\frac{1}{2}\left[\mathscr{P}_{A},[\Omega, \Omega]\right]=0 .
$$

The second term in (B13) vanishes if $U_{A B}^{\gamma}$ are constants. For our purposes, we are interested in the situation where these functions actually vanish. In this case, the first term also vanishes and we therefore find

$$
\left[\hat{H}_{A}, \hat{H}_{B}\right]=0 \text {. }
$$

This result is crucial for our representation of $\operatorname{diff}(M)$ on $\hat{\Gamma}^{\prime}$ and $\hat{\Gamma}^{\prime \prime}$ in Sec. $V$.

\section{APPENDIX C: NOTATION AND CONVENTIONS}

\section{A. Indices}

Latin indices from the beginning of the alphabet $(a, b, c$, etc.) are abstract indices for tensors on $M$. Two-dimensional coordinate basis indices are denoted by Greek letters from the beginning of the alphabet ( $\alpha, \beta, \gamma$, etc.); they take the values zero and 1 . Indices for preferred coordinates associated with a choice of gauge are further distinguished by parentheses $[(\alpha),(\beta)$, etc. ]. General target space indices are denoted as upper case Latin letters. When we have a flat Minkowski target space, as for the string, we use Greek letters from the latter part of the alphabet to denote components with respect to an inertial coordinate frame. The spacelike directions orthogonal to a pair of null directions in the target Minkowski space are labeled by the Latin indices $i$, $j, k$, etc.

\section{B. Coordinates}

Generic coordinates on $M$ are denoted $\mathrm{X}^{\alpha}$. Preferred coordinates are labeled $Y^{(\alpha)}$. Coordinates on $R \times S^{1}$ are denoted as $\sigma^{\alpha}=(\tau, \sigma)$ where $\tau \in(-\infty, \infty)$ and $\sigma \in[0,2 \pi]$, with $\sigma=0$ and $\sigma=2 \pi$ identified.

\section{Metrics and derivative operators}

We use $g_{a b}$ to denote a metric on $M$ : It has the signature $(-+)$. Note that this metric is a priori independent of the induced metric on the string world sheet. The two-dimensional Minkowski metric in inertial coordinates is denoted $\eta_{\alpha \beta}$. The general target space metric is denoted $G_{A B}$. The 
target space Minkowski metric in inertial coordinates is denoted $\eta_{\mu v}$. The signature here is $(-++\cdots+)$. We use the symbols $\gamma$ and $\tilde{\gamma}$ to represent one-dimensional metrics induced on spacelike embeddings of circles in $M$.

The derivative operator $\nabla_{a}$ on $M$ is torsion-free and compatible with $g_{a b}$. The Lie derivative with respect to the vector $V^{a}$ is denoted $\mathscr{L}_{V}$. We use $\partial_{1}$ and the associated comma notation to denote partial derivatives on the circle with respect to the coordinate $\sigma$.

\section{Groups and Lie algebras}

The diffeomorphism group of the manifold $M$ is denoted $\operatorname{Diff}(M)$. The generic element is $\phi \in \operatorname{Diff}(M)$. The associated Lie algebra is $\operatorname{diff}(M)$, with the typical elements $U, V$, $W \in \operatorname{diff}(M)$. Similarly, the group of conformal isometries of the metric $g_{a b}$ on $M$ is denoted $\operatorname{Conf}(M, g)$, with the generic element $\psi \in \operatorname{Conf}(M, g)$. The Lie algebra of $\operatorname{Conf}(M, g)$ is $\operatorname{conf}(M, g)$, with the typical elements $v, w \in \operatorname{conf}(M, g)$.

\section{E. Deformation vectors}

Unless otherwise stated, the symbol $N^{\alpha}$ stands for an externally prescribed (" $c$ number") smearing field. The lapse function $N^{\perp}$ and shift vector $N^{1}$, obtained by projecting $N^{\alpha}$, are, respectively, a scalar function and a vector on $S^{1}$. The symbol $N$ denotes the lapse density, which is $N^{\downarrow}$ rescaled to be a density of weight minus 1 on the circle. Smearing fields that are obtained by restriction of a space-time vector $V^{a}$ to an embedding retain the same root letter; these fields are functionals of the embeddings (i.e., " $q$ numbers").

\section{F. Phase spaces}

We use $\Gamma$ to denote the usual string phase space. Its slight extension, as defined in Sec. IV, is denoted $\Gamma^{*}$. We obtain $\Gamma^{\prime}$ by adding to $\Gamma$ the embedding phase space in conjunction with the conformal gauge. We obtain $\Gamma^{\prime \prime}$ by adding embedding and Lagrange multiplier phase spaces to $\Gamma$ in conjunction with the harmonic gauge. We denote the phase space for harmonic maps from $M$ into $M^{d}$ by $\Gamma_{0}$ and $\Gamma_{0}^{\prime}$ is its embedding-extended counterpart. A caret (e.g., $\widehat{\Gamma}$ ) over any of the above phase spaces denotes their BRST extensions. An overbar (e.g., $\bar{\Gamma}$ ) denotes the submanifold obtained by imposing all relevant constraints.

\section{G. Brackets}

The symbol [,] is used to represent both Poisson brackets and vector field commutators. Which meaning is intended in a given expression should be clear from the context.

\section{H. The symbol $X$}

The symbol $X$ is used in a variety of ways and its meaning should be clear from the context in which it is being used. An embedding of a circle into $M$ is denoted abstractly as $X$. Its coordinate form is $X^{\alpha}(\sigma)$. A one-parameter family of such embeddings, i.e., a foliation of $M$, is also denoted abstractly as $X$ : Its coordinate form is $X^{\alpha}(\sigma, \tau)$. As described above, $\mathrm{X}^{\alpha}$ represent generic coordinates on $M$.

\footnotetext{
'See, e.g., A. J. Hanson, T. Regge, and C. Teitelboim, Constrained Hamiltonian Systems (Academia Nazionale dei Lincei, Rome, 1976); K. Sundermeyer, Constrained Dynamics (Springer, New York, 1982) and references therein.

${ }^{2}$ C. J. Isham and K. V. Kuchă, Ann. Phys. (NY) 164, 288, 316 (1985).

${ }^{3}$ A. Polyakov, Phys. Lett. B 103, 207, 211 (1981).

${ }^{4}$ See, e.g., M. Green, J. Schwarz, and E. Witten, Superstring Theory (Cambridge U.P., Cambridge, 1987); L. Brink and M. Henneaux, Principles of String Theory (Plenum, New York, 1988) and references therein.

${ }^{5}$ K. V. Kuchar̆ and C. G. Torre, "Strings as Poor Relatives of Relativity," University of Utah preprint (1988).

${ }^{6} \mathrm{~K}$. V. Kuchar̆, J. Math. Phys. 13, 768 (1972).

${ }^{7}$ L. Balieu, W. Siegel, and B. Zwiebach, Nucl.Phys. B 287, 93 (1987).

${ }^{8}$ P. A. M. Dirac, Lectures on Quantum Mechanics (Yeshiva Univ., New York, 1964).

${ }^{9}$ P. A. M. Dirac, Proc. R. Soc. London Ser. A 246, 333 (1958); R.Arnowitt, S. Deser, and C. W. Misner, in Gravitation: An Introduction to Current Research, edited by L.Witten (Wiley, New York, 1962).

${ }^{10}$ K. V. Kuchař, J. Math. Phys. 19, 390 (1978).

"M. Bowick and S. G. Rajeev, Phys. Rev. Lett. 58, 535 (1987).

${ }^{12}$ A. Patracioiu, Nucl. Phys. B 81, 525 (1974); see, also, the second reference of Ref. 4.

${ }^{13}$ M. Henneaux, Phys. Rep. 126, 1 (1985).

${ }^{14}$ D. McMullan and J. Paterson, Phys. Lett. B 202, 358 (1988).

${ }^{15}$ S. R. Das and M. A. Rubin, Phys. Lett. B 169, 182 (1986).

${ }^{16} \mathrm{~K}$. V. Kuchař and C. G. Torre, in preparation.

${ }^{17} \mathrm{~K}$. V. Kuchař, "Parametrized Scalar Field on $R \times S^{1}$ : Dynamical Pictures, Space-time Diffeomorphisms and Conformal Isometries" and "The Dirac Constraint Quantization of a Parametrized Field Theory by Anomaly-Free Operator Representations of Spacetime Diffeomorphisms," University of Utah preprints (1988).

${ }^{18}$ E. S. Fradkin and G. A. Vilkovisky, CERN Rep. TH2332 (1977); I. A. Batalin and G. A. Vilkovisky, Phys. Lett. B 69, 309 (1977).
} 